\title{
Synthesis of Novel 2-(Het)arylpyrrolidine Derivatives and Evaluation of Their Anticancer and Anti-Biofilm Activity
}

 \\ Elena Chugunova 1,4,*(D), Ivan Sazykin ${ }^{2}$, Anastasiya Gildebrant ${ }^{2}$ (D), Julia Voronina ${ }^{5}$, \\ Alexander Burilov ${ }^{1}$, Shorena Karchava ${ }^{2}{ }^{\mathbb{D}}$, Maria Klimova ${ }^{2}$, Alexandra Voloshina ${ }^{1}$, \\ Anastasia Sapunova ${ }^{1}$, Elena Klimanova ${ }^{6}$, Tatyana Sashenkova ${ }^{6}$, Ugulzhan Allayarova ${ }^{6}$, \\ Anastasiya Balakina ${ }^{6,7}$ and Denis Mishchenko ${ }^{6,7}$ (D) \\ 1 Arbuzov Institute of Organic and Physical Chemistry, FRC Kazan Scientific Center of RAS, Arbuzov str., 8, \\ Kazan 420088, Russia \\ 2 Southern Federal University, Stachki Avenue, 194/2, Rostov-on-Don 344090, Russia \\ 3 Institute of Chemical Research and Technology of Korkyt Ata Kyzylorda State University, Aiteke bie str., \\ 29A, Kyzylorda 120014, Kazakhstan \\ 4 Kazan Federal University, Kremlyovskaya str., 18, Kazan 420008, Russia \\ 5 N. S. Kurnakov Institute of General and Inorganic Chemistry, RAS, 31 Leninsky Av., Moscow 119991, Russia \\ 6 Institute of Problems of Chemical Physics RAS, Chernogolovka 142432, Russia \\ 7 Scientific and Educational Center in Chernogolovka of Moscow Region State University, Mytishi 141014, \\ Russia \\ * Correspondence: agazizov@iopc.ru (A.G.); chugunova.e.a@gmail.com (E.C.); \\ Tel.: +7-843-272-7324 (A.G.); +7-843-272-7324 (E.C.)
}

Academic Editors: Carla Boga and Gabriele Micheletti

Received: 22 July 2019; Accepted: 22 August 2019; Published: 25 August 2019

\begin{abstract}
A library of novel 2-(het)arylpyrrolidine-1-carboxamides were obtained via a modular approach based on the intramolecular cyclization/Mannich-type reaction of $N$-(4,4-diethoxybutyl)ureas. Their anti-cancer activities both in vitro and in vivo were tested. The in vitro activity of some compounds towards M-Hela tumor cell lines was twice that of the reference drug tamoxifen, whereas cytotoxicity towards normal Chang liver cell did not exceed the tamoxifen toxicity. In vivo studies showed that the number of surviving animals on day 60 of observation was up to $83 \%$ and increased life span (ILS) was up to $447 \%$. Additionally, some pyrrolidine-1-carboxamides possessing a benzofuroxan moiety obtained were found to effectively suppress bacterial biofilm growth. Thus, these compounds are promising candidates for further development both as anti-cancer and anti-bacterial agents.
\end{abstract}

Keywords: pyrrolidine; carboxamide; anti-tumor activity; anti-cancer activity; cytotoxicity; apoptosis; bacterial biofilm; anti-bacterial activity

\section{Introduction}

The pyrrolidine moiety is an important structural part of many natural alkaloids [1-4] and one of the most frequently occurring heterocyclic scaffolds in approved drugs [5]. A number of anti-cancer drugs possess an $\mathrm{N}$-carboxypyrrolidine scaffold. These include both fairly old ones (e.g., dactinomycin [6], approved in 1964) and those that have appeared recently. Acalabrutinib [7,8] (approved by FDA in 2017) and larotrectinib [9,10] (approved by the FDA in 2018) may serve as illustrative examples (Figure 1). Notably, larotrectinib is the first drug to be specifically developed and approved to treat any cancer containing certain mutations, as opposed to cancers of specific tissues. It should also be emphasized that both acalabrutinib and larotrectinib contain a 2-(het)aryl-substituted 
pyrrolidine fragment. Considering that in the past few decades, cancer has been one of major causes of death in most countries of the world [11], a search for novel anti-cancer drug candidates among 2-(het)aryl-N-carboxypyrrolidine derivatives is undoubtedly a promising field of research.<smiles>CC#CC(=O)N1CCC[C@H]1c1nc(-c2ccc(C(=O)Nc3ccccn3)cc2)c2c(N)nccn12</smiles>

Figure 1. Approved anti-cancer drugs possessing 2-substituted N-carboxypyrrolidine scaffold.

Despite the increasing number of research works aimed at obtaining and studying 2-(hetaryl)pyrrolidines, the synthesis of these compounds still meets certain difficulties. Approaches to these compounds can be divided into two main groups. The first one includes the modification of an existing pyrrolidine fragment. Various cross-coupling reactions of (hetero)aromatics with appropriately substituted pyrrolidine derivatives [12-17], including oxidative [18-20] and photooxidative ones [21-24], are the most often used within this pathway. In several cases, the synthesis of enantiomerically pure 2-(het)arylpyrrolidines has been also accomplished by decarboxylative (hetero)arylation of proline derivatives [25-31]. The second approach is based on the formation of a pyrrolidine ring from acyclic precursors. Within this approach, the intermolecular [3+2] dipolar cycloaddition of activated alkenes to azomethine ylides plays a significant role [32-37]. Essential drawbacks of the abovementioned approaches are the need of expensive metal catalysts and/or harsh reaction conditions, as well as the need of the preliminary synthesis of starting compounds with appropriate functional groups and desired fragments. Hence, methods employing inexpensive, readily available reagents and catalysts and allowing simultaneous pyrrolidine ring closure and $\mathrm{C}-\mathrm{C}_{\text {hetaryl }}$ bond formation are of a special interest.

Earlier, we developed a metal-free approach to 2-arylsubstitued pyrrolidine derivatives based on the acid-catalyzed intramolecular cyclization of $N$-(4,4-diethoxybutyl)ureas, leading to the formation of a pyrrolinium cation. Further Mannich-type reaction of this cyclic iminium ion with various electron-rich aromatic C-nucleophiles allowed us to obtain a range of 2-arylpyrrolidine-1-carboxamides [38-45]. The main benefits of this approach are the usage of easily accessible starting materials and its modularity, which allows wide variability in both the aromatic moiety and substituents at the nitrogen atom (Scheme 1). 


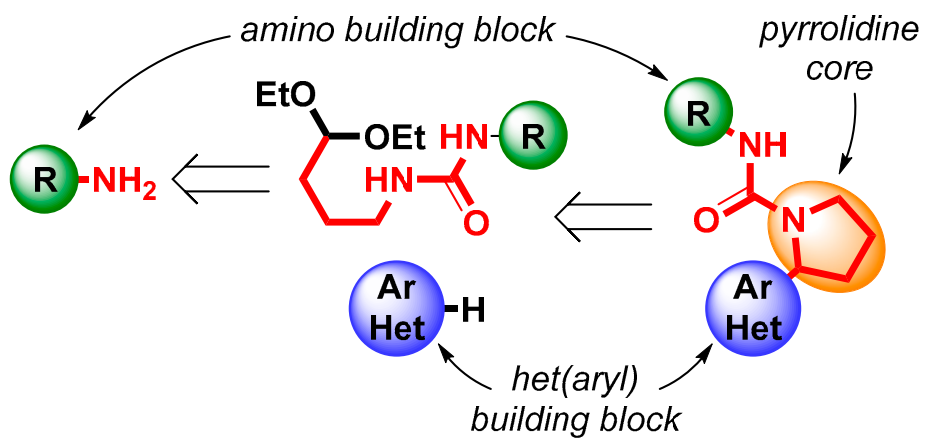

Scheme 1. Modular synthetic approach to 2-(het)arylpyrrolidine-1-carboxamides.

Herein, we report the successful extension of this approach to the synthesis of novel 2-(het)aryl-substituted pyrrolidine-1-carboxamides, as well as the evaluation of their anti-cancer activities in vivo and in vitro and studies of the inhibition of bacterial biofilm growth.

\section{Results and Discussion}

\subsection{Chemistry}

We started our research from the synthesis of initial $\mathrm{N}$-(4,4-diethoxybutyl)ureas $\mathbf{1}$ by the previously described procedure $[38,46]$. Both aliphatic amines and substituted anilines were employed as the amine building block (see Scheme 1). It is well known that the ionization of an amine group is widely used for drug solubility enhancement $[47,48]$. Thus, $N$-(4,4-diethoxybutyl)urea $\mathbf{1 h}$ possessing a dimethylamino moiety was also obtained (Table 1 ).

Table 1. Synthesized library of novel (het)arylpyrrolidines $5-\mathbf{8}^{1}$.

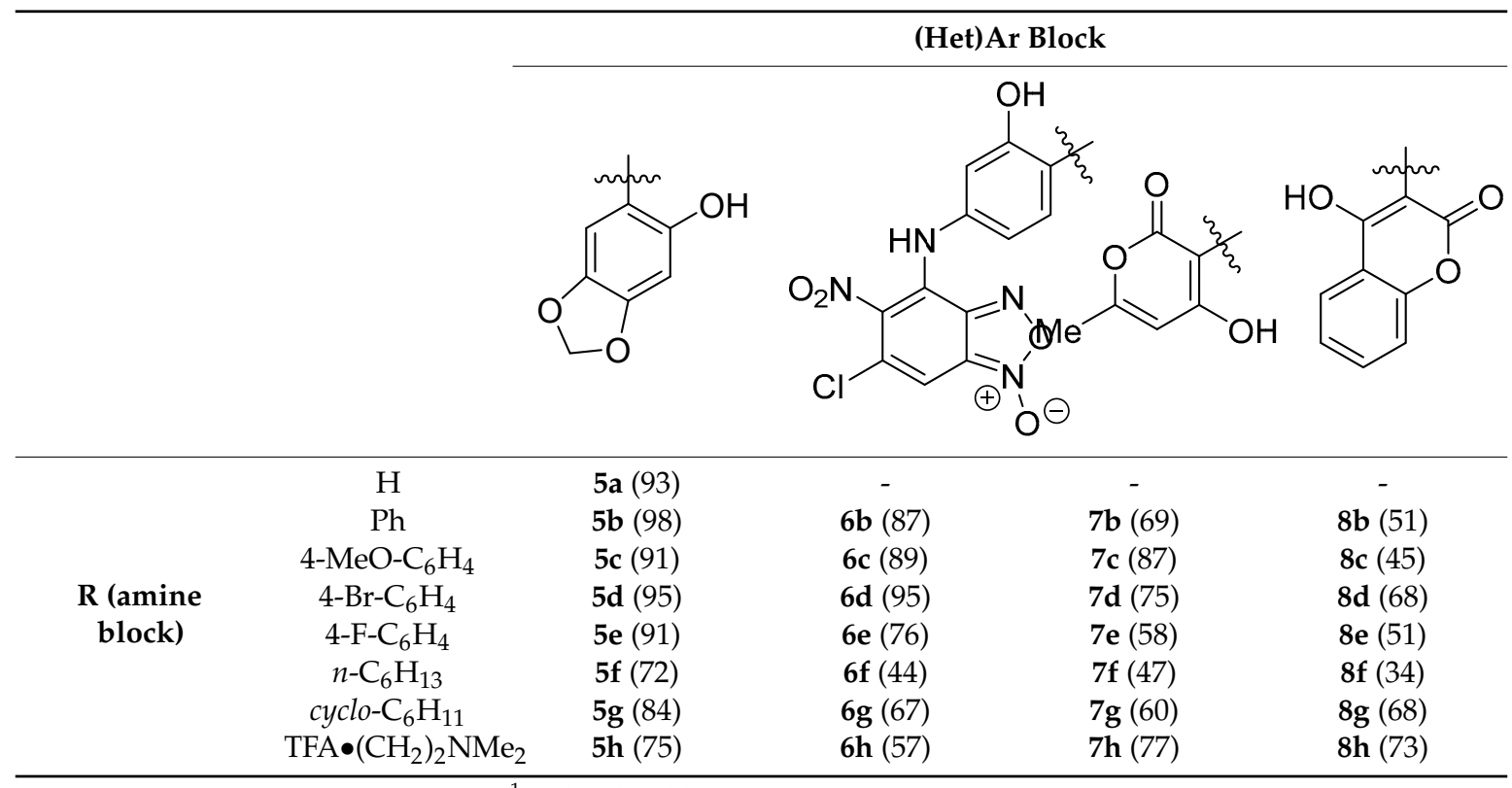

${ }^{1}$ Isolated yields are given in parentheses.

The choice of aromatic (sesamol [49,50]) and heterocyclic (4-hydroxycoumarin [51], 4-hydroxy-6-methyl-2H-pyran-2-one [52,53]) C-nucleophiles was determined by both their well-known biological activity and high reactivity in electrophilic substitution reactions. One more heterocyclic scaffold, namely, the benzofuroxan moiety, was also of interest due to its biological properties [54-56] and ability to serve as NO donor $[57,58]$. However, it could not be introduced to the target pyrrolidines 
directly due to its extremely low nucleophilicity. Thus, the phenol derivative 4 was used instead, which was obtained by the reaction of 4,6-dichloro-5-nitrobenzofuroxan 2 with 3-aminophenol 3 (Scheme 2).

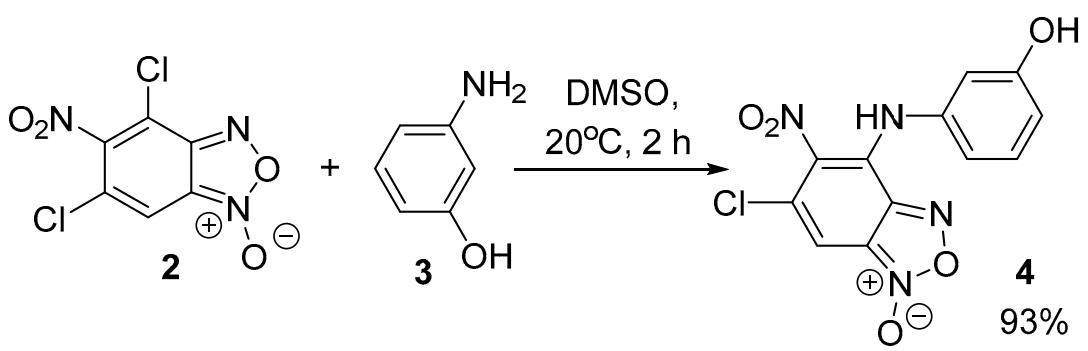

Scheme 2. Synthesis of substituted phenol 4 possessing a benzofuroxan fragment.

Next, we carried out the reaction of ureas $\mathbf{1 a - h}$ with these $C$-nucleophiles in the presence of trifluoroacetic acid as catalyst (Scheme 3). As a result, a library of 28 novel 2-(het)arylpyrrolidine-1-carboxamides $5 \mathbf{b}-\mathbf{h}, \mathbf{6 b}-\mathbf{h}, \mathbf{7 b}-\mathbf{h}, \mathbf{8 b}-\mathbf{h}$ was obtained, which included all possible combinations of amine and (het)aryl building blocks. Additionally, the non-substituted pyrrolidine-1-carboxamide 5a was also obtained. Yields of target compounds varied from moderate to excellent (Table 1). The substitution sites of the used C-nucleophiles were confirmed by NMR data. The structure of compound $\mathbf{8 f}$ was additionally confirmed by X-ray analysis data.

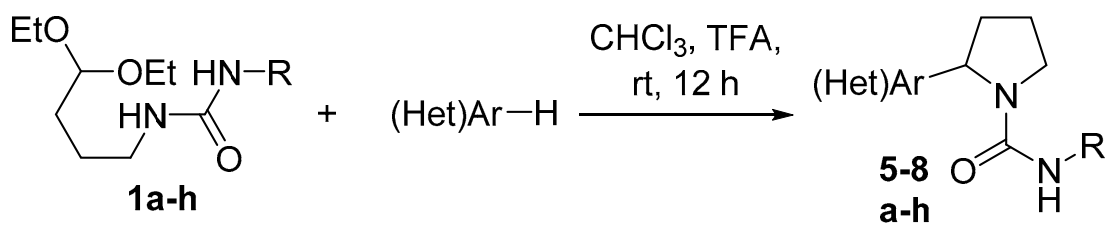

Scheme 3. Synthesis of (het)arylpyrrolidine-1-carboxamides 5-8.

Compound $8 f$ crystallized with three independent molecules in the unit cell. Bond lengths, valence, and torsion angles were within the intervals typical for each bond type (Figure 2A). Molecules $a, b$, and $c$ differed in the conformations of the pyrrolidine cycle (envelope in each case, however in molecules a and c atom C3 was out of the plane formed by other atoms, while in molecule b it was atom C4 and hexane substituent (Supplementary Materials, Table S1). Crystal packing consisted of centrosymmetric H-bonded dimers in which each independent molecule interacted only with its symmetric equivalent (Figure 2A, Supplementary Materials, Table S2). Dimers formed columns via $\pi \cdots \pi$ interactions (Supplementary Materials, Table S3). The three-dimensional system (Supplementary Materials, Figure S15) was formed by weaker CH‥O (Supplementary Materials, Table S1) and CH $\cdots \pi$ interactions (Supplementary Materials, Table S4). 


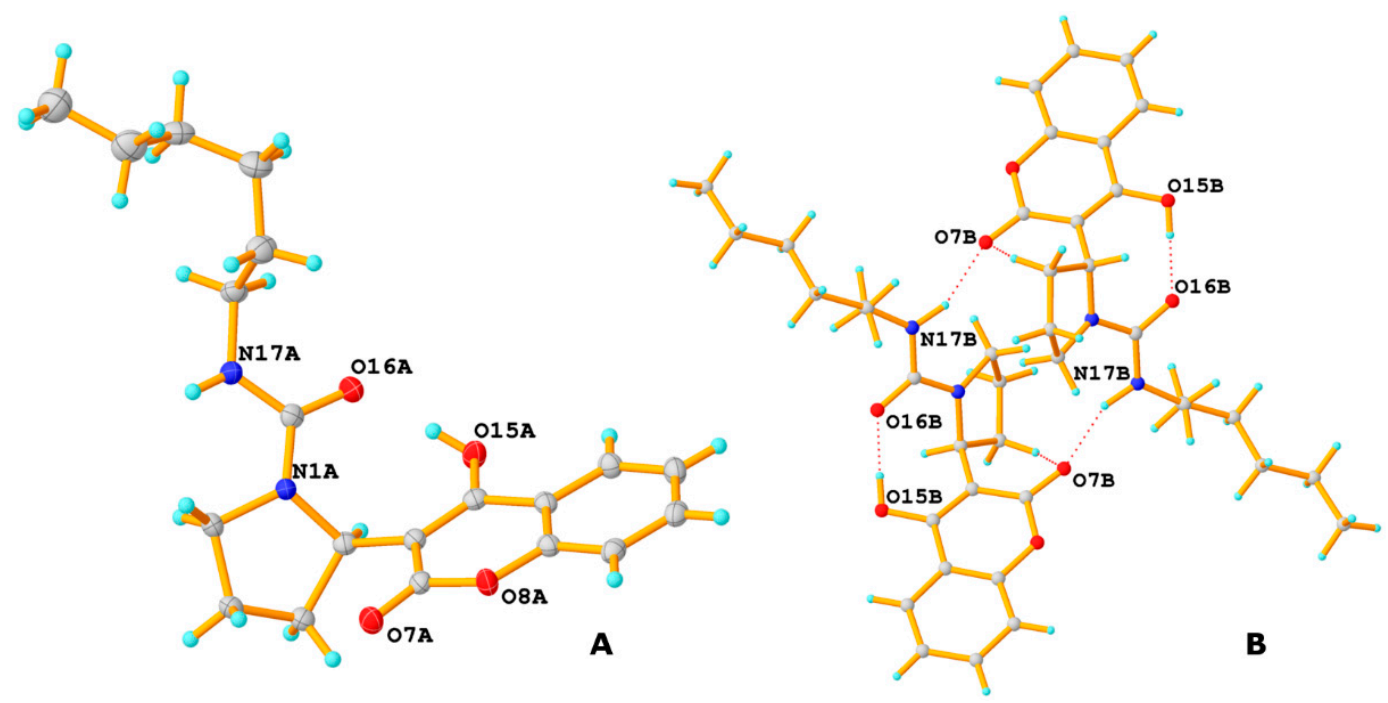

Figure 2. (A) Molecular structure of compound $\mathbf{8 f}$ in crystal (on the example of molecule a). Ellipsoids are shown with $50 \%$ probability; (B) H-bonded dimer in crystal of $\mathbf{8 f}$ (on the example of molecule b).

\subsection{Biological Studies}

\subsubsection{In Vitro Studies of Anti-Cancer Activity}

Cytotoxic assay. The resulting compounds were tested for cytotoxicity against normal and cancerous human cell lines at concentrations of $1-100 \mu \mathrm{M}$. The pyrrolidines $\mathbf{6 b}-\mathbf{h}$ containing the benzofuroxane fragment were found to be the most active, while the others $(5 \mathbf{a}-5 \mathbf{h}, 7 \mathbf{b}-7 \mathbf{h}, \mathbf{8 b}-\mathbf{8 h})$ did not show anti-cancer activity (Table 2, Supplementary Materials, Table S5). Table 2 shows the $\mathrm{IC}_{50}$ data for compounds $\mathbf{6 b}-\mathbf{h}$. It can be seen that in relation to the M-Hela cancer line, $\mathrm{IC}_{50}$ values for substances $\mathbf{6 d}, \mathbf{6 c}$, and $\mathbf{6 e}$ were comparable to the reference compound tamoxifen. Compound $\mathbf{6 g}\left(\mathrm{IC}_{50}\right.$ $-14.7 \mu \mathrm{M})$ was most active against cervical cancer. At the same time, with regard to the Chang's liver cell line, all test compounds were less toxic than tamoxifen.

Table 2. Cytotoxic effects of pyrrolidines $5 \mathbf{a}-\mathbf{6 h}$ on the cancer and normal human cell lines ${ }^{1}$.

\begin{tabular}{|c|c|c|c|c|c|}
\hline \multirow{3}{*}{$\begin{array}{c}\text { Test } \\
\text { Compound }\end{array}$} & \multicolumn{2}{|c|}{$\mathrm{IC}_{50}(\mu \mathrm{M})$} & \multirow{3}{*}{$\begin{array}{c}\text { Test } \\
\text { Compound }\end{array}$} & \multicolumn{2}{|c|}{$\mathrm{IC}_{50}(\mu \mathrm{M})$} \\
\hline & $\begin{array}{c}\text { Cancer Cell } \\
\text { Line }\end{array}$ & $\begin{array}{c}\text { Normal Cell } \\
\text { Line }\end{array}$ & & $\begin{array}{c}\text { Cancer Cell } \\
\text { Line }\end{array}$ & $\begin{array}{c}\text { Normal Cell } \\
\text { Line }\end{array}$ \\
\hline & M-Hela & Chang Liver & & M-Hela & Chang Liver \\
\hline $5 a$ & $>100$ & $>100$ & - & - & - \\
\hline $5 b$ & $>100$ & $>100$ & $6 b$ & $56 \pm 4.1$ & $>100$ \\
\hline $5 c$ & $>100$ & $>100$ & $6 c$ & $26.0 \pm 1.9$ & $62 \pm 4.3$ \\
\hline $5 d$ & $>100$ & $>100$ & $6 d$ & $25.5 \pm 1.6$ & $53 \pm 3.8$ \\
\hline $5 e$ & $>100$ & $>100$ & $6 e$ & $26 \pm 1.8$ & $48 \pm 3.0$ \\
\hline $5 f$ & $>100$ & $>100$ & $6 f$ & $47 \pm 2.9$ & $57 \pm 4.3$ \\
\hline $5 g$ & $>100$ & $>100$ & $6 \mathrm{~g}$ & $14.7 \pm 0.9$ & $46 \pm 2.7$ \\
\hline $5 \mathrm{~h}$ & $>100$ & $>100$ & $6 h$ & $100 \pm 8.6$ & $>100$ \\
\hline & & & tamoxifen & $28.0 \pm 2.5$ & $46.2 \pm 3.5$ \\
\hline
\end{tabular}

${ }^{1}$ Three independent experiments were carried out.

Induction of apoptotic effects by test compounds. For compound $6 \mathrm{~g}$, the ability to induce apoptosis in the human cancer cell line M-Hela was studied. As shown in Figure 3 (bottom), after 24 hours of treatment with compound $\mathbf{6 g}$, apoptotic effects were observed in M-Hela cells (red fluorescence). The data presented in Figure 3 show that at concentrations corresponding to the $\mathrm{IC}_{50}$ value, compound $6 \mathrm{~g}$ induced apoptosis in 25\% of M-Hela cells. The top row of images in Figure 3 (control) presents 
images of intact M-Hela cells. It is seen that apoptotic effects were weakly expressed. The results show that the cytotoxicity of compound $\mathbf{6} \mathbf{g}$ in M-Hela cancer cells was caused by an apoptotic pathway.
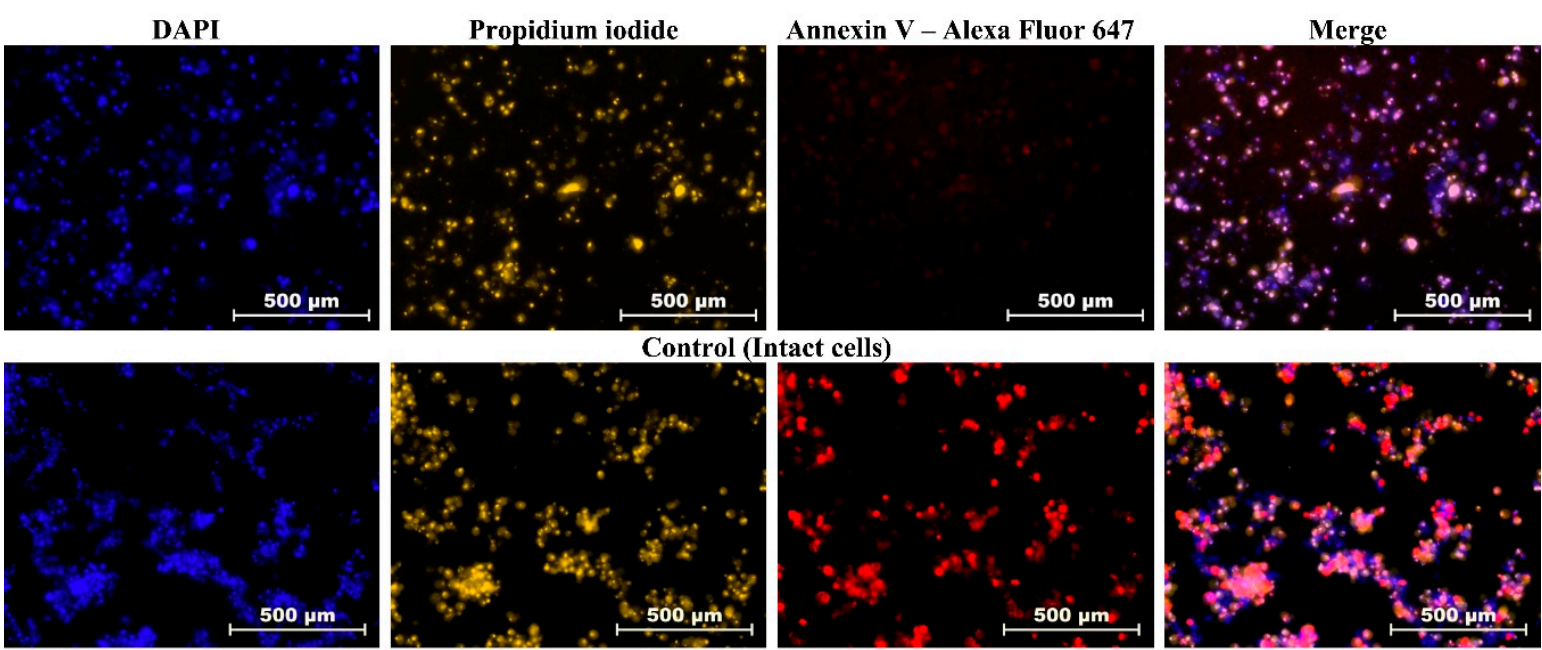

$6 \mathrm{~g}(\mathrm{IC50}-14.7 \mu \mathrm{M})$

Figure 3. Images of control intact M-Hela cells (top) and M-Hela cells after treatment with $\mathbf{6 g}$ at an $\mathrm{IC}_{50}$ concentration of $14.7 \mu \mathrm{M}$ (bottom), obtained using the Cytell Cell Imaging multifunctional system using the BioApp Automated Imaging application. Annexin V-Alexa Fluor 647 (red fluorescence) was used to detect apoptotic cells; living cells-DAPI (blue fluorescence); dead cells-propidium iodide (yellow fluorescence).

Multiplex analysis of early apoptosis markers. Next, using the MILLIPLEX ${ }^{\circledR}$ MAP 7-plex Early Phase Apoptosis Signaling kit, seven markers of early apoptosis of JNK, Bad, Bcl-2, Akt, Caspase-9, p53, and Caspase-8 were detected in M-Hela cell lysates. The median fluorescence intensity (MFI) was measured using the Luminex ${ }^{\circledR}$ system. This assay is a quick and convenient alternative to Western blot and immunoprecipitation.

Figure 4 shows that the fluorescence intensity of Caspase- 8 in the experimental sample (after exposure to the test substance $\mathbf{6 g}$ was two times higher than that in the control. The results suggest that apoptosis proceeded along the extrinsic pathway of activation of Caspase-8 (death is initiated by activation of the surface cell receptor), and not along the intrinsic pathway associated with the activation of Caspase-9 (fluorescence intensity at the control level) in which death occurs due to mitochondrial dysfunction. This assumption was also confirmed by the predominance of pro-apoptotic Bad proteins over anti-apoptotic Bcl-2, which are responsible for irreversible cellular damage in mitochondrial processes. At the same time, apoptosis in M-Hela cells can be induced by activating the transcription of many pro-apoptotic genes by the transcription factors AP1 (signaling pathway activated by JNK stress) and p53 (response to DNA damage).

Effects on the mitochondrial membrane potential $(\Delta \psi \mathrm{m})$ by lead compounds. In confirmation of data on the course of apoptosis in cells along an external pathway not associated with dysfunctional mitochondria, the ability of the tested compounds to reduce the potential of the mitochondrial membrane $(\Delta \psi \mathrm{m})$ in M-Hela culture cells was examined using the example of compound $6 \mathrm{~g}$. Studies were performed using flow cytometry methods using JC-10 reagent. In normal cells with a high potential of the mitochondrial membrane, the dye JC-10 forms aggregates (J-aggregate) near the mitochondrial membranes. When the membrane potential due to the stimulation of apoptosis falls, JC-10 is evenly distributed in the cell as a monomer (J-monomer). JC-10 units in normal cells have red fluorescence, and JC-10 monomers are green. The ratio between orange-red and green fluorescence can be used to assess the onset of apoptosis. No decrease in $\Delta \psi \mathrm{m}$ was demonstrated using flow cytometry analysis (Figure 5). The intensity of red fluorescence after treating cells with compound $\mathbf{6 g}$ in an $\mathrm{IC}_{50}$ concentration of $14.7 \mu \mathrm{M}$ did not actually change compared with the control. The results obtained indicate that the 
mechanism of action of the studied compounds is not associated with the induction of apoptosis, which proceeds along the mitochondrial pathway.

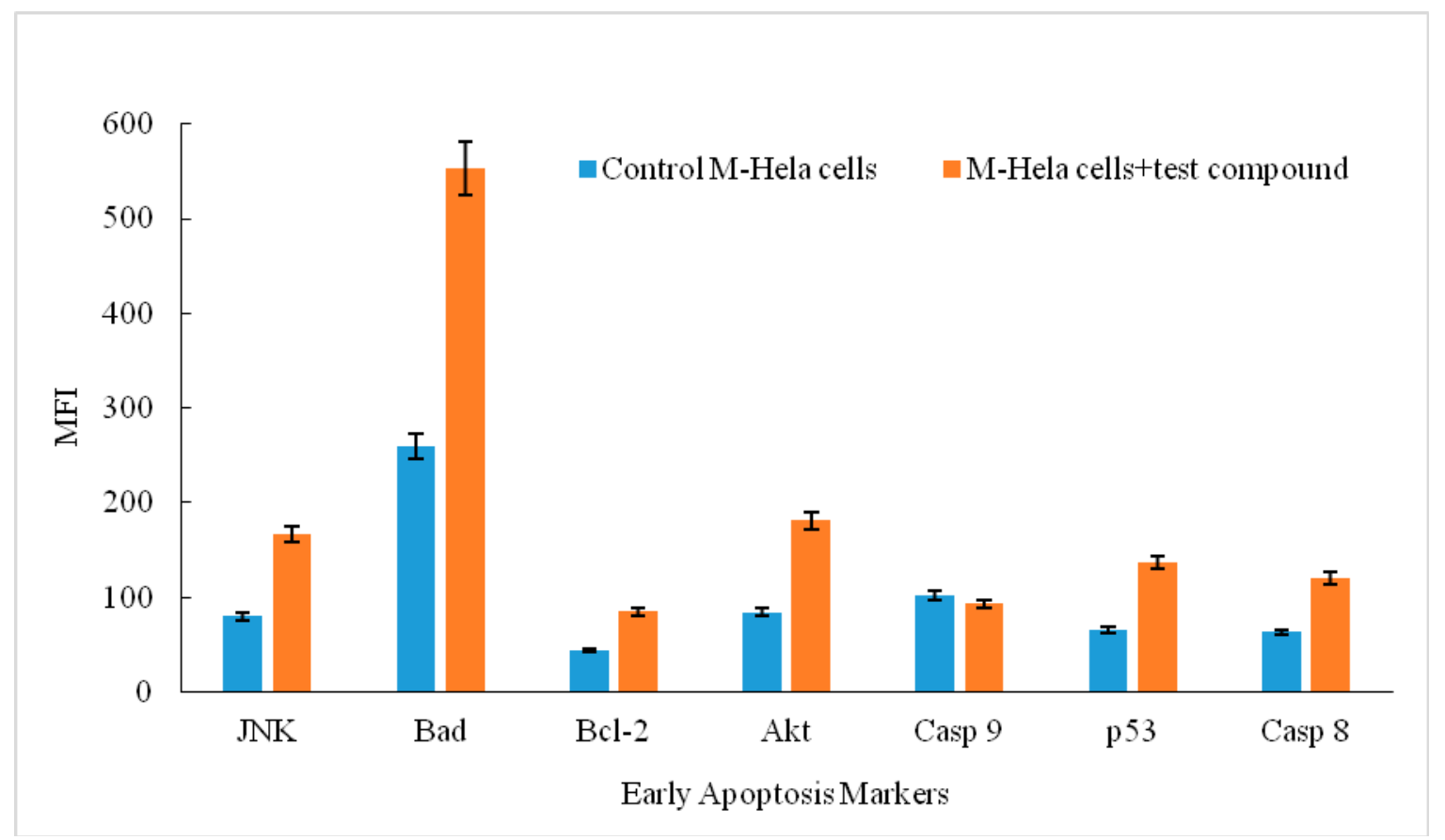

Figure 4. Multiplex analysis of early apoptosis markers in M-Hela cells treated with the test substance $6 \mathrm{~g}$ at an $\mathrm{IC}_{50}$ concentration of $14.7 \mu \mathrm{M}$ as well as M-Hela cells untreated with the test substance (control). The median fluorescence intensity (MFI) was measured using the Luminex ${ }^{\circledR}$ system. The graph shows the mean and standard deviation values for the wells in triplicate.
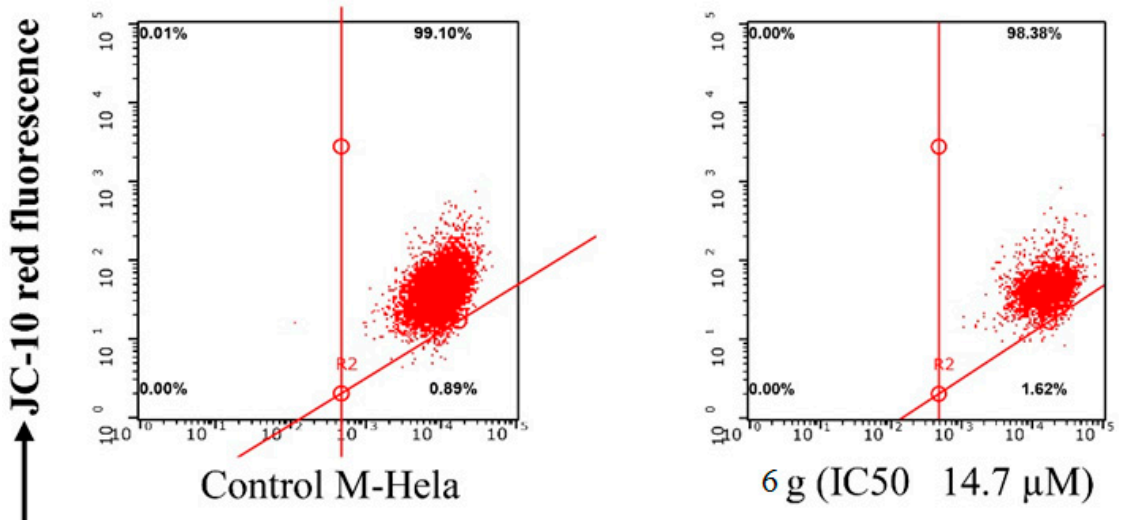

\section{JC-10 green fluorescence}

Figure 5. Flow cytometry analysis of M-Hela cells treated with compound $6 \mathrm{~g}$, along with the quantification of $\%$ of cells with red aggregates. The values are presented as mean $\pm \mathrm{SD}$.

\subsubsection{In Vivo Studies of Anti-Cancer Activity}

In vivo evaluation was performed on the syngeneic P388 murine leukemia. Murine leukemia models have been an essential component of the initial drug discovery programs since the 1970s. P388 leukemia played a major role in the screening of potential antitumor agents. Today, the majority of currently used clinical drugs were first detected by the murine leukemias. These models are suitable for the initial evaluation of the antitumor activity of new compounds [59]. The compounds were administered i.p. (thus, as an intra-tumor treatment), which was believed to maximize exposure and 
limit pharmacokinetic influences. Due to the fact that as a solvent for parenteral administration of the substance it is permissible to use any physiologically appropriate solvent that does not cause local irritating effects such as water or saline [60], we selected only water-soluble compounds $5 \mathbf{b}, \mathbf{5 h}, \mathbf{6 h}$, and $7 \mathrm{~h}$ for in vivo studies.

We found that the compounds $5 \mathbf{b}, 5 \mathbf{h}$, and $\mathbf{8 h}$ had pronounced anti-leukemic activity with the number of surviving animals on day 60 of observation from $17 \%$ to $83 \%$ and increased life span (ILS) from $80 \%$ to $447 \%$ (Table 3, Table 4, and Supplementary Materials, Figure S16). Compound 5 h had the greatest anti-leukemic activity. Indeed, in the group of mice that received intraperitoneal administration of compound $5 \mathrm{~h}$ at a dose of $40 \mathrm{mg} / \mathrm{kg} /$ day, 1, 5, and 9 days after tumor transplantation, $83 \%$ of the animals remained alive, and ILS was $447 \%$. In general, the studied compounds can be arranged in the following order of reducing the antitumor activity during therapy of P388 leukemia: $5 \mathrm{~h}>8 \mathrm{~h}>5 \mathrm{~b}$. However, compounds $6 \mathrm{~h}$ and $7 \mathrm{~h}$ in the dose range and the mode of administration used did not show antitumor properties. Thus, $\mathbf{5 b}, \mathbf{5 h}$, and $\mathbf{8 h}$ can be recommended as promising compounds for the creation of new anticancer drugs.

Table 3. Mean survival time and increased life span (ILS) of murine leukemia P388 at individual treatment with $5 \mathrm{~h}, 8 \mathrm{~h}, 5 \mathrm{~b}, 6 \mathrm{~h}$, and $7 \mathrm{~h}$.

\begin{tabular}{|c|c|c|c|c|c|c|c|c|c|c|}
\hline \multirow{2}{*}{ Compound } & \multicolumn{10}{|c|}{ Dose } \\
\hline & $\begin{array}{c}\mathrm{MST} \pm \mathrm{SD}^{1} \\
\text { (days) }\end{array}$ & $\begin{array}{c}\text { ILS }^{2} \\
(\%)\end{array}$ & $\begin{array}{c}\mathrm{MST} \pm \mathrm{SD}^{1} \\
\text { (days) }\end{array}$ & $\begin{array}{c}\text { ILS }^{2} \\
(\%)\end{array}$ & $\begin{array}{l}\mathrm{MST} \pm \mathrm{SD}^{1} \\
\text { (days) }\end{array}$ & $\begin{array}{c}\text { ILS }^{2} \\
(\%)\end{array}$ & $\begin{array}{c}\mathrm{MST} \pm \mathrm{SD}^{1} \\
\text { (days) }\end{array}$ & $\begin{array}{c}\text { ILS }^{2} \\
(\%)\end{array}$ & $\begin{array}{c}\text { MST } \pm \text { SD }^{1} \\
\text { (days) }\end{array}$ & $\begin{array}{c}\text { ILS }^{2} \\
(\%)\end{array}$ \\
\hline $5 b$ & $27.7 \pm 10.3^{* * *}$ & 177 & $\underset{* * * *}{26.5 \pm 10.6}$ & 165 & $26.0 \pm 10.8^{* *}$ & 160 & $18.0 \pm 8.4^{*}$ & 80 & $9.7 \pm 0.2$ & 0 \\
\hline $5 \mathrm{~h}$ & $35.7 \pm 10.9^{* * *}$ & 276 & $18.5 \pm 8.3$ * & 95 & $52.0 \pm 8.0 * * *$ & 447 & $38.0 \pm 9.9^{* * *}$ & 300 & $19.0 \pm 8.2^{* *}$ & 100 \\
\hline $7 \mathrm{~h}$ & $11.0 \pm 0.6^{*}$ & 0 & $10.3 \pm 0.2$ & 0 & $10.2 \pm 0.2$ & 0 & $10.7 \pm 0.2$ & 0 & $10.3 \pm 0.2$ & 0 \\
\hline $8 \mathrm{~h}$ & $19.3 \pm 8.1^{*}$ & 93 & $20.7 \pm 8.0^{* *}$ & 107 & $13.2 \pm 1.6^{* * *}$ & 32 & $19.8 \pm 8.1^{* *}$ & 98 & $26.2 \pm 10.7^{* *}$ & 162 \\
\hline
\end{tabular}

${ }^{1}$ MST: mean survival time; SD: standard deviation; ${ }^{2}$ ILS $\%$ : the percentage of the median survival time (MST) of the treated group $(\mathrm{t})$ to that of the control group (c). ILS \% $=(\mathrm{MSTt} / \mathrm{MST}) \times 100$. ${ }^{*}$ Statistically significant increase over the control $(p<0.05) ; * *$ Statistically significant increase over the control $(p<0.01) ; * *$ Statistically significant increase over the control $(p<0.001)$. 
Table 4. Effect of compounds $5 \mathbf{b}, 5 \mathbf{h}, \mathbf{6 h}, \mathbf{7 h}$, and $8 \mathrm{~h}$ against i.p. implanted P388 murine tumors.

\begin{tabular}{|c|c|c|c|c|c|c|c|c|c|c|}
\hline \multirow{3}{*}{ Compound } & \multicolumn{10}{|c|}{ Dose } \\
\hline & \multicolumn{2}{|c|}{$18 \mathrm{mg} / \mathrm{kg} / \mathrm{day}$} & \multicolumn{2}{|c|}{$26 \mathrm{mg} / \mathrm{kg} / \mathrm{day}$} & \multicolumn{2}{|c|}{$40 \mathrm{mg} / \mathrm{kg} / \mathrm{day}$} & \multicolumn{2}{|c|}{$55 \mathrm{mg} / \mathrm{kg} / \mathrm{day}$} & \multicolumn{2}{|c|}{$83 \mathrm{mg} / \mathrm{kg} / \mathrm{day}$} \\
\hline & Survivors/Total ${ }^{1}$ & Survival, $\%$ & Survivors/Total ${ }^{1}$ & Survival, $\%$ & Survivors/Total ${ }^{1}$ & Survival, $\%$ & Survivors/Total ${ }^{1}$ & Survival, $\%$ & Survivors/Total ${ }^{1}$ & Survival, $\%$ \\
\hline Control & $0 / 6$ & 0 & $0 / 6$ & 0 & $0 / 6$ & 0 & $0 / 6$ & 0 & $0 / 6$ & 0 \\
\hline $5 b$ & $2 / 6$ & 33 & $2 / 6$ & 33 & $2 / 6$ & 33 & $2 / 6$ & 17 & $0 / 6$ & 0 \\
\hline $5 \mathrm{~h}$ & $3 / 6$ & 50 & $1 / 6$ & 17 & $5 / 6$ & 83 & $3 / 6$ & 50 & $1 / 6$ & 17 \\
\hline $6 \mathrm{~h}$ & $0 / 6$ & 0 & $0 / 6$ & 0 & $0 / 6$ & 0 & $0 / 6$ & 0 & $0 / 6$ & 0 \\
\hline $7 \mathrm{~h}$ & $0 / 6$ & 0 & $0 / 6$ & 0 & $0 / 6$ & 0 & $0 / 6$ & 0 & $0 / 6$ & 0 \\
\hline $8 \mathrm{~h}$ & $1 / 6$ & 17 & $1 / 6$ & 17 & $1 / 6$ & 0 & $1 / 6$ & 17 & $1 / 6$ & 33 \\
\hline
\end{tabular}

${ }^{1}$ Survivors on day 60. 


\subsubsection{Anti-Biofilm Activity of Pyrrolidine-1-Carboxamides Possessing Benzofuroxan Moiety}

Biofilms are communities of microorganisms attached to the surface or the interface in which cells are immersed in an exopolymer matrix consisting of polysaccharides, proteins, and DNA [61]. More than $90 \%$ of microorganisms occurring in nature exist in the form of biofilms [62]. Microbial biofilms are responsible for the etiology and pathogenesis of many acute and especially chronic bacterial infections in humans [63]. Among bacterial diseases in humans and animals, more than $80 \%$ are associated with the presence of stable bacterial communities enclosed in biofilms [64,65].

The possibility of interspecies community formation in biofilms along with high antibiotic resistance of many pathogenic and potentially pathogenic microorganisms make them almost invulnerable. The drug resistance of bacteria living in biofilms has increased manyfold in comparison to planktonically grown bacteria [66,67]. In this regard, the ability of pathogenic bacteria to form biofilms is a significant problem.

Moreover, in around $20 \%$ of all cases, microbial organisms are the causative agents of cancer-inducing inflammation. It is unclear if these microorganisms are causally involved in tumorigenesis, or if they benefit from the consequences of tumor growth and in turn promote tumor progression [68,69]. Furthermore, the work of Samanta et al. [70] demonstrates that the mechanisms behind advanced anti-biofilm and anticancer activities are linked to the generation of excess labile toxic reactive oxygen species (ROS). Such toxic ROS species cause the rapid oxidation and deterioration of cellular membranes. The unity of the action mechanism possibly testifies to the interconnection of anti-biofilm and anticancer activities in certain substances. Taking this into account, we also evaluated the ability of pyrrolidine-1-carboxamides $6 \mathbf{a}-\mathbf{e}$ possessing a benzofuroxan moiety and the initial benzofuroxan 4 to inhibit bacterial biofilm growth.

A staining assay was performed to estimate the extent of biofilm formation by Vibrio aquamarinus DSM 26054 and Acinetobacter calcoaceticus VKPM B-10353 under treatment with 6a, 6b, 6c, 6d, 6e, and 4 (doses of $1 \times 10^{-9}-1 \times 10^{-5} \mathrm{M}$ ). The natural strains A. calcoaceticus VKPM B-10353 and V. aquamarinus DSM 26054 were chosen as models due to their ability to actively form biofilms and for their extreme degree of similarity to pathogenic species. The obtained biological activity results are summarized in Table 5 (see Supplementary Materials, Figures S1-S14 for additional data). The compounds were evaluated for biofilm production compared to control. The results showed that compounds exhibited a variable degree of anti-biofilm activity against $V$. aquamarinus DSM 26054 and $A$. calcoaceticus VKPM B-10353.

Table 5. Biofilm formation (\%) by Vibrio aquamarinus DSM 26054 and Acinetobacter calcoaceticus VKPM B-10353 in the presence of pyrrolidine-1-carboxamides possessing benzofuroxan moiety in reference to control (control $=100 \%$ ).

\begin{tabular}{|c|c|c|c|c|c|c|c|}
\hline \multirow[b]{2}{*}{ № } & \multirow[b]{2}{*}{ Compound } & \multirow[b]{2}{*}{ Strain } & \multirow{2}{*}{\multicolumn{5}{|c|}{$\begin{array}{c}\text { Biofilm Formation, } \% \\
\text { Compound Concentration, } \mathrm{M}\end{array}$}} \\
\hline & & & & & & & \\
\hline 1 & $6 \mathbf{a}$ & V. aquamarinus DSM 26054 & $17.70 *$ & $10.23 *$ & $20.34^{*}$ & $16.49^{*}$ & $42.65^{*}$ \\
\hline \multirow{2}{*}{2} & \multirow{2}{*}{$6 b$} & V. aquamarinus DSM 26054 & $8.21 *$ & $15.42 *$ & $26.04 *$ & $39.02 *$ & 89.12 \\
\hline & & A. calcoaceticus VKPM B-10353 & 90.41 & $70.79^{*}$ & $73.93 *$ & 88.44 & $119.69^{*}$ \\
\hline 3 & $6 c$ & V. aquamarinus DSM 26054 & 13.54 * & $14.67^{*}$ & $9.22 *$ & $18.28^{*}$ & $26.65^{*}$ \\
\hline 4 & $6 d$ & A. calcoaceticus VKPM B-10353 & $79.16^{*}$ & $46.95^{*}$ & $50.00 *$ & 95.71 & 89.50 \\
\hline \multirow[t]{2}{*}{5} & \multirow[t]{2}{*}{$6 e$} & V. aquamarinus DSM 26054 & 67.29 & $17.96^{*}$ & $11.01 *$ & $26.02 *$ & $39.57 *$ \\
\hline & & A. calcoaceticus VKPM B-10353 & $87.92 *$ & $47.66^{*}$ & $55.40^{*}$ & $53.27^{*}$ & 86.50 \\
\hline \multirow{2}{*}{6} & \multirow[t]{2}{*}{4} & V. aquamarinus DSM 26054 & 66.58 & $9.31 *$ & 11.34 * & $43.56^{*}$ & 96.57 \\
\hline & & A. calcoaceticus VKPM B-10353 & $86.67^{*}$ & $62.12^{*}$ & $78.63^{*}$ & 97.99 & $149.22^{*}$ \\
\hline 7 & Azithromycin & V. aquamarinus DSM 26054 & 103.42 & 102.43 & 99.39 & 103.80 & $81.50 *$ \\
\hline
\end{tabular}

* Differences compared to the control samples are statistically significant, $t$ criterion, $p<0,05$; the solutions of appropriate solvent in ethanol with the same concentration were used as control in experiments with pyrrolidine-1-carboxamides; six replicates were done for each treatment and control. 
Substances $\mathbf{6 b}$ and 4 had different effects on A. calcoaceticus VKPM B-10353 biofilm formation-both suppressing and stimulating, depending on the concentration. Due to the dual nature of their action, they are not recommended for use as agents suppressing biofilm development.

For substances $\mathbf{6 a}, \mathbf{6 d}, \mathbf{6 c}$, and $\mathbf{6 e}$, the suppressive effect of different values on the formation of biofilm strains V. aquamarinus DSM 26054 and A. calcoaceticus VKPM B-10353 was registered in the range of investigated concentrations. Biofilm formation in comparison with control varied from $6.46 \%$ to $87.92 \%$.

Biofilm formation by A. calcoaceticus VKPM B-10353 was less suppressed in the presence of substances than biofilm formation by $V$. aquamarinus DSM 26054. Biofilm formation varied from $42.37 \%$ to $87.92 \%$ with respect to control in the presence of the studied substances. For the V.aquamarinus DSM 26054 strain, it ranged from $6.46 \%$ to $67.29 \%$.

Substances actively inhibited the growth of $A$. calcoaceticus VKPM B-10353 biofilms at the concentrations of $1 \times 10^{-8} \mathrm{M}$ and $1 \times 10^{-7} \mathrm{M}$. The maximum inhibition of biofilms was registered under the influence of $6 \mathbf{a}$ and $6 \mathbf{d}$ at the concentration of $1 \times 10^{-8} \mathrm{M}$, and biofilm formation was $42.37 \%$ and $46.95 \%$, respectively.

The maximum inhibition of $V$. aquamarinus DSM 26054 biofilm was caused by $\mathbf{6 a}$ and $\mathbf{6 d}$ at the concentration of $1 \times 10^{-8} \mathrm{M}$ (biofilm formation was $10.23 \%$ and $6.46 \%$ in comparison with the control) and $6 \mathrm{c}$ and $6 \mathrm{e}$ at the concentration of $1 \times 10^{-7} \mathrm{M}$ (biofilm formation amounted to $9.22 \%$ and $11.01 \%$ ).

Note that $\mathbf{6 a}$ and $\mathbf{6 c}$ actively suppressed the formation of $V$. aquamarinus DSM 26054 biofilm at the minimum concentration of $1 \times 10^{-9} \mathrm{M}$ - the preservation of biofilm was $17.7 \%$ and $13.54 \%$, respectively.

The tested compounds were also compared with standard antibiotics azithromycin (see Supplementary Materials for additional data). Azithromycin exhibited an insignificant suppression of biofilm at high concentrations. Azithromycin suppressed the intensity of biofilm formation by $V$. aquamarinus DSM 26054 at the concentration of $1 \times 10^{-5} \mathrm{M}$ (Supplementary Materials, Figure S13). The optical density was $81.5 \%$ of the control values. The inhibitory effect of azithromycin in the studied concentrations on biofilm formation by A. calcoaceticus VKPM B-10353 was not detected (Supplementary Materials, Figure S14).

Taken together, pyrrolidine-1-carboxamides $\mathbf{6 a}, \mathbf{6 c}, \mathbf{6 d}$, and $\mathbf{6 e}$ possessed a greater potential to suppress the formation of biofilms compared to the initial substance, as well as to the antibiotic azithromycin, and are promising as agents suppressing biofilms.

Genotoxicity and pro-oxidant characteristics of pyrrolidine-1-carboxamides possessing benzofuroxan moiety. All compounds in the same concentrations used for biofilm attenuation were also tested with Escherichia coli MG1655 (pRecA-lux). This strain was used for the evaluation of genotoxicity (Table 6). Bioluminescent response to DNA damage was detected for compounds $\mathbf{6 b}, \mathbf{6 c}$, and $\mathbf{6 e}$. The detected genotoxic effect was evaluated as medium (I > 2) for $\mathbf{6 b}, \mathbf{6 c}$, and $\mathbf{6 e}$ in the concentration range of $10^{-9}-10^{-5} \mathrm{M}$, and as weak $(\mathrm{I}<2)$ for $6 \mathbf{b}$ at the concentration of $10^{-5} \mathrm{M}$. These compounds are direct mutagens. Substance $\mathbf{6 c}$, in addition, is also a promutagen. Its genotoxicity was registered under conditions of metabolic activation in the concentration range of $10^{-7}-10^{-6} \mathrm{M}$. Compounds $\mathbf{6 a}, \mathbf{6 d}$, and 4 do not belong to the class of DNA-damaging substances. 
Table 6. Genotoxicity (induction factor, I) of the pyrrolidine-1-carboxamides possessing a benzofuroxan moiety registered with the bacterial lux-biosensor Escherichia coli MG1655 (pRecA-lux).

\begin{tabular}{|c|c|c|c|c|c|c|}
\hline \multirow{2}{*}{ Compound } & \multirow{2}{*}{ Activation $^{1}$} & \multicolumn{5}{|c|}{ Concentration of Compound, $\mathrm{M}$} \\
\hline & & $10^{-9}$ & $10^{-8}$ & $10^{-7}$ & $10^{-6}$ & $10^{-5}$ \\
\hline \multirow{2}{*}{$6 a$} & - & $0.65 \pm 0.01$ & $0.88 \pm 0.06$ & $0.88 \pm 0.03$ & $0.61 \pm 0.03$ & $0.54 \pm 0.01$ \\
\hline & + & $0.72 \pm 0.04$ & $0.86 \pm 0.01$ & $0.80 \pm 0.03$ & $0.70 \pm 0.05$ & $0.65 \pm 0.06$ \\
\hline \multirow{2}{*}{$6 b$} & - & $2.34 \pm 0.06^{*}$ & $2.28 \pm 0.12 *$ & $2.07 \pm 0.11 *$ & $2.15 \pm 0.06^{*}$ & $1.77 \pm 0.04$ * \\
\hline & + & $1.47 \pm 0.04$ & $1.31 \pm 0.01$ & $1.30 \pm 0.02$ & $1.36 \pm 0.04$ & $1.23 \pm 0.02$ \\
\hline \multirow{2}{*}{$6 c$} & - & $2.67 \pm 0.07 *$ & $2.84 \pm 0.02 *$ & $2.64 \pm 0.14$ * & $2.82 \pm 0.05 *$ & $2.33 \pm 0.04$ * \\
\hline & + & $1.30 \pm 0.01$ & $1.37 \pm 0.02$ & $1.49 \pm 0.02$ * & $1.54 \pm 0.03$ * & $1.35 \pm 0.02$ \\
\hline \multirow{2}{*}{$6 d$} & - & $0.64 \pm 0.03$ & $0.70 \pm 0.01$ & $0.62 \pm 0.03$ & $0.70 \pm 0.01$ & $0.60 \pm 0.02$ \\
\hline & + & $0.76 \pm 0.05$ & $0.79 \pm 0.03$ & $0.71 \pm 0.05$ & $0.71 \pm 0.03$ & $0.66 \pm 0.04$ \\
\hline \multirow{2}{*}{$6 e$} & - & $2.56 \pm 0.10 *$ & $2.74 \pm 0.04$ * & $2.46 \pm 0.02 *$ & $2.39 \pm 0.02 *$ & $2.00 \pm 0.02$ * \\
\hline & + & $1.43 \pm 0.01$ & $1.35 \pm 0.04$ & $1.45 \pm 0.02$ & $1.47 \pm 0.04$ & $1.24 \pm 0.02$ \\
\hline \multirow{2}{*}{4} & - & $1.21 \pm 0.04$ & $1.27 \pm 0.07$ & $1.14 \pm 0.02$ & $1.27 \pm 0.01$ & $1.10 \pm 0.00$ \\
\hline & + & $1.19 \pm 0.01$ & $1.11 \pm 0.02$ & $1.18 \pm 0.01$ & $1.28 \pm 0.04$ & $1.11 \pm 0.01$ \\
\hline
\end{tabular}

${ }^{1}$ Variants with metabolic activation (+S9) and without it (-S9); ${ }^{*}$ difference from the control experiment are statistically significant, $t$-test; $p<0.05$

Prooxidant characteristics (production of superoxide anion and NO) were evaluated using the biosensor E. coli MG1655 (pSoxS-lux) (Table 7). Compound 6b did not possess prooxidant activity. A weak response was observed for compounds $6 \mathbf{a}\left(10^{-9}-10^{-8} \mathrm{M}\right), \mathbf{6 d}\left(10^{-6} \mathrm{M}\right), \mathbf{6 c}\left(10^{-8}-10^{-7} \mathrm{M}\right)$, and $6 \mathbf{e}\left(10^{-7} \mathrm{M}\right)$. On the other hand, for compound 4, a significant effect of superoxide-anion radical or NO level increase was registered in a bacterial cell at the concentration of $1 \times 10^{-8} \mathrm{M}$, and a weak effect was seen for concentrations $10^{-9}-10^{-8} \mathrm{M}$ and $10^{-7}-10^{-5} \mathrm{M}$.

Table 7. Prooxidant activity (induction factor) of the pyrrolidine-1-carboxamides possessing a benzofuroxan moiety registered with the bacterial lux-biosensor E. coli MG1655 (pSoxS-lux).

\begin{tabular}{cccccc}
\hline \multirow{2}{*}{ Compound } & \multicolumn{5}{c}{ Concentration of Compound, M } \\
\cline { 2 - 5 } & $\mathbf{1 0}^{\mathbf{- 9}}$ & $\mathbf{1 0}^{-\mathbf{8}}$ & $\mathbf{1 0}^{-\mathbf{7}}$ & $\mathbf{1 0}^{-\mathbf{6}}$ & $\mathbf{1 0}^{-\mathbf{5}}$ \\
\hline $\mathbf{6 a}$ & $1.54 \pm 0.13^{*}$ & $1.70 \pm 0.23^{*}$ & $1.36 \pm 0.03$ & $1.46 \pm 0.06$ & $1.24 \pm 0.08$ \\
\hline $\mathbf{6 b}$ & $1.03 \pm 0.10$ & $1.21 \pm 0.00$ & $1.04 \pm 0.04$ & $1.24 \pm 0.11$ & $0.89 \pm 0.03$ \\
\hline $\mathbf{6 c}$ & $1.11 \pm 0.03$ & $1.54 \pm 0.20^{*}$ & $1.68 \pm 0.20 *$ & $1.46 \pm 0.03$ & $0.96 \pm 0.09$ \\
\hline $\mathbf{6 d}$ & $1.34 \pm 0.09$ & $1.11 \pm 0.07$ & $1.11 \pm 0.01$ & $1.60 \pm 0.23 *$ & $1.10 \pm 0.07$ \\
\hline $\mathbf{6 e}$ & $0.93 \pm 0.03$ & $1.25 \pm 0.28$ & $1.85 \pm 0.15^{*}$ & $1.34 \pm 0.10$ & $0.84 \pm 0.04$ \\
\hline $\mathbf{4}$ & $1.85 \pm 0.10 *$ & $2.08 \pm 0.05 *$ & $1.74 \pm 0.14 *$ & $1.54 \pm 0.07 *$ & $1.64 \pm 0.04{ }^{*}$ \\
\hline
\end{tabular}

${ }^{*}$ Difference from the control experiment are statistically significant, $t$-test; $p<0.05$.

Taken together, the most promising candidates for further studies are compounds $\mathbf{6 a}$ and $\mathbf{6} \mathbf{d}$, for which maximum suppression of bacterial biofilm growth was observed. These compounds were found to be non-genotoxic and possessed a weak pro-oxidant activity. However, a careful study of their biological activity in eukaryotic models is still required. 


\section{Materials and Methods}

\subsection{Chemistry}

IR spectra were recorded on a UR-20 spectrometer in the $400-3600 \mathrm{~cm}^{-1}$ range in $\mathrm{KBr} .{ }^{1} \mathrm{H}-\mathrm{NMR}$ spectra were recorded on a Bruker AVANCE $400(400 \mathrm{MHz})$ spectrometer (Bruker BioSpin, Rheinstetten, Germany) with respect to the signals of residual protons of deuterated solvent $\left(\mathrm{CDCl}_{3}, \mathrm{DMSO}-\mathrm{d}_{6}\right)$. ${ }^{13} \mathrm{C}-\mathrm{NMR}$ spectra were recorded on a Bruker Avance $600(151 \mathrm{MHz})$ spectrometer (Bruker BioSpin, Rheinstetten, Germany) relative to signals of residual protons of deuterated solvent $\left(\mathrm{CDCl}_{3}, \mathrm{DMSO}-\mathrm{d}_{6}\right)$. Elemental analysis was performed on a CHNS-O Elemental Analyser EuroEA3028-HT-OM (EuroVector S.p.A., Milan, Italy). The melting points were determined in glass capillaries on a Stuart SMP 10 instrument. $N$-(4,4-diethoxybutyl)ureas 1a-g were obtained as previously described $[38,46]$.

The X-ray diffraction data for crystal of compound $8 \mathbf{f}$ were collected at $150 \mathrm{~K}$ on a Bruker AXS Smart Apex II CCD diffractometer in the $\omega$ and $\varphi$ scan modes using graphite monochromated MoK $\alpha$ $(\lambda 0.71073 \AA$ A $)$ radiation. The structure was solved by direct method and refined by the full matrix least-squares using the SHELXTL program [71]. All non-hydrogen atoms were refined anisotropically. The positions of hydrogen atoms were located from the Fourier electron density synthesis and were included in the refinement in the isotropic riding model approximation. All figures were made using OLEX2 [72] and Mercury [73]. Crystallographic data for the structure reported in this paper have been deposited with the Cambridge Crystallographic Data Center (1941912, www.ccdc.ac.uk).

6-Chloro-4-((3-hydroxyphenyl)amino)-5-nitrobenzo[c][1,2,5]oxadiazole 1-oxide (4). To the solution of benzofuroxan $3(0.40 \mathrm{~g}, 1.6 \mathrm{mmol})$ in DMSO $(3 \mathrm{~mL})$ a solution of 3-aminophenol $(0.35 \mathrm{~g}, 3.2 \mathrm{mmol})$ in DMSO $(3 \mathrm{~mL})$ was added at room temperature. The reaction mixture was stirred at room temperature for $2 \mathrm{~h}$, reagents consumption was monitored by TLC (eluent: toluene/ethylacetate, 2/1). Then the reaction mixture was poured in water $(100 \mathrm{~mL})$, precipitate was filtered off, washed with water, and dried. Crude product was purified by column chromatography (eluent: toluene/ethylacetate, 2/1) and recrystallized from chloroform/hexane (3/1) to give target compound 4 as dark solid. Yield $93 \%$, m.p. 128-130 ${ }^{\circ} \mathrm{C}$; IR ( $\left.v, \mathrm{~cm}^{-1}\right): 1563,1628,3094,3320,3447 ;{ }^{1} \mathrm{H}-\mathrm{NMR}\left(400 \mathrm{MHz}, \mathrm{CHCl}_{3}, \delta \mathrm{ppm}\right) 4.91(\mathrm{~s}, 1 \mathrm{H}$, NH), 6.73-6.75 (m, 1H, Ar-H), 6.80-6.84 (m, 2H, Ar-H), 6.92 (s, 1H, Ar-H), 7.27 (s, 1H, Ar-H), 8.49 (s, $1 \mathrm{H}, \mathrm{OH}) ;{ }^{13} \mathrm{C}-\mathrm{NMR}\left(151 \mathrm{MHz}, \mathrm{CHCl}_{3}, \delta \mathrm{ppm}\right)$ 102.7, 111.8, 113.0, 114.8, 117.1, 128.4, 130.4, 130.6, 132.6, 138.8, 146.1, 156.3; Elemental analysis: calc. for $\mathrm{C}_{12} \mathrm{H}_{7} \mathrm{ClN}_{4} \mathrm{O}_{5}$ (322.5): $\mathrm{C} 44.67 ; \mathrm{H} 2.19 ; \mathrm{Cl} 10.99 ; \mathrm{N} 17.36$; found C 44.49; $\mathrm{H} 2.32 ; \mathrm{Cl} 10.83 ; \mathrm{N}$ 17.44. ESI $\mathrm{m} / z$ : $[\mathrm{M}+\mathrm{H}]^{+}$: calc. for $\mathrm{C}_{12} \mathrm{H}_{8} \mathrm{ClN}_{4} \mathrm{O}_{5}$ 323; found 323 .

1-(4,4-Diethoxybutyl)-3-(2-(dimethylamino)ethyl)urea (1h). To a solution of $N^{1}, N^{1}$-dimethylethane-1,2 -diamine $(0.97 \mathrm{~g}, 11.0 \mathrm{mmol})$ in dichloromethane $(11 \mathrm{~mL}) 1,1^{\prime}$-carbonyldiimidazole $(2.0 \mathrm{~g}, 12.3 \mathrm{mmol})$ was added. The reaction mixture was stirred for 48 hours at room temperature. Then 4,4-diethoxybutan-1-amine $(1.77 \mathrm{~g}, 11.0 \mathrm{mmol})$ was added and reaction mixture was stirred for another $48 \mathrm{~h}$ at room temperature. The reaction mixture was extracted with water $(3 \times 10 \mathrm{~mL})$, the organic layer was separated, and solvent was removed in vacuum to give target compound $1 \mathrm{~h}$ as yellow oil. Yield 64\%; ${ }^{1} \mathrm{H}-\mathrm{NMR}\left(400 \mathrm{MHz}, \mathrm{CHCl}_{3}, \delta \mathrm{ppm}\right) 1.10\left(\mathrm{t}, 6 \mathrm{H}, \mathrm{J}=7.1 \mathrm{~Hz}, \mathrm{CH}_{3}\right), 1.40-1.49(\mathrm{~m}$, $\left.2 \mathrm{H}, \mathrm{CH}_{2}\right), 1.53-1.60\left(\mathrm{~m}, 2 \mathrm{H}, \mathrm{CH}_{2}\right), 2.13\left(\mathrm{~s}, 6 \mathrm{H}, \mathrm{CH}_{3}\right), 2.31\left(\mathrm{t}, 2 \mathrm{H}, \mathrm{J}=5.9 \mathrm{~Hz}, \mathrm{CH}_{2}\right), 3.04-3.11(\mathrm{~m}, 2 \mathrm{H}$, $\left.\mathrm{CH}_{2}\right), 3.12-3.20\left(\mathrm{~m}, 2 \mathrm{H}, \mathrm{CH}_{2}\right), 3.36-3.45\left(\mathrm{~m}, 2 \mathrm{H}, \mathrm{CH}_{2}\right), 3.51-3.60\left(\mathrm{~m}, 2 \mathrm{H}, \mathrm{CH}_{2}\right), 4.39(\mathrm{t}, 1 \mathrm{H}, J=5.6 \mathrm{~Hz}$, $\mathrm{CH}), 5.37(\mathrm{~s}, 1 \mathrm{H}, \mathrm{NH}), 5.55(\mathrm{~s}, 1 \mathrm{H}, \mathrm{NH}) ;{ }^{13} \mathrm{C}-\mathrm{NMR}\left(151 \mathrm{MHz}, \mathrm{CHCl}_{3}, \delta \mathrm{ppm}\right)$ 15.2, 25.5, 31.1, 38.1, 40.0, $45.2,59.3,61.2,102.8,159.1$.

General method for the synthesis of pyrrolidine-1-carboxamides 5-8. To a mixture of appropriate C-nucleophile $(1.61 \mathrm{mmol})$ and chloroform $(5 \mathrm{~mL})$, urea $1(1.61 \mathrm{mmol})$ and trifluoroacetic acid $(0.18 \mathrm{~g}$, $1.61 \mathrm{mmol} ; 0.36 \mathrm{~g}, 3.22 \mathrm{mmol}$ in the case of urea $\mathbf{1 h}$ ) were added. The reaction mixture was stirred for $24 \mathrm{~h}$ at room temperature, the solvent was removed in vacuum, and the residue was washed thoroughly with diethyl ether and dried in vacuum to give title compound.

2-(6-Hydroxybenzo[d][1,3]dioxol-5-yl)pyrrolidine-1-carboxamide (5a). Beige solid, yield 93\%, m.p. 206-207 ${ }^{\circ} \mathrm{C}$; IR $\left(v, \mathrm{~cm}^{-1}\right): 1534,1637,2986,3174,3294 ;{ }^{1} \mathrm{H}-\mathrm{NMR}\left(400 \mathrm{MHz}, \mathrm{DMSO}-d_{6}, \delta \mathrm{ppm}\right) 1.69-1.77$ $\left(\mathrm{m}, 1 \mathrm{H}, \mathrm{CH}_{2}\right), 1.78-1.88\left(\mathrm{~m}, 2 \mathrm{H}, \mathrm{CH}_{2}\right), 2.06-2.16\left(\mathrm{~m}, 1 \mathrm{H}, \mathrm{CH}_{2}\right), 3.29-3.36\left(\mathrm{~m}, 1 \mathrm{H}, \mathrm{CH}_{2}\right), 3.46-3.52(\mathrm{~m}$, 
$\left.1 \mathrm{H}, \mathrm{CH}_{2}\right), 4.93-4.99(\mathrm{~m}, 1 \mathrm{H}, \mathrm{CH}), 5.48-5.80\left(\mathrm{br} \mathrm{s}, 2 \mathrm{H}, \mathrm{NH}_{2}\right), 5.86\left(\mathrm{dd}, 2 \mathrm{H}, J=4.3 \mathrm{~Hz}, 1.0 \mathrm{~Hz}, \mathrm{CH}_{2}\right)$, ), 6.41 (s, 1H, Ar-H), 6.47 (s, 1H, Ar-H); 9.07-9.86 (br s, 1H, OH). ${ }^{13} \mathrm{C}-\mathrm{NMR}$ (151 MHz, DMSO- $\left.d_{6}, \delta \mathrm{ppm}\right)$ 23.8, 33.2, 46.9, 55.3, 98.4, 100.9, 106.2, 122.9, 140.1, 146.3, 149.1, 157.9; Elemental analysis: calc. for $\mathrm{C}_{12} \mathrm{H}_{14} \mathrm{~N}_{2} \mathrm{O}_{4}(250)$ : C, 57.59; $\mathrm{H}, 5.64 ; \mathrm{N}, 11.19$; found C, 57.70; H, 5.71; N, 11.01; ESI m/z: $[\mathrm{M}+\mathrm{H}]^{+}$: calc. for $\mathrm{C}_{12} \mathrm{H}_{15} \mathrm{~N}_{2} \mathrm{O}_{4} 251$; found 251 .

2-(6-Hydroxybenzo[d][1,3]dioxol-5-yl)-N-phenylpyrrolidine-1-carboxamide (5b). Beige solid, yield 98\%, m.p. $190-191{ }^{\circ} \mathrm{C}$; IR $\left(v, \mathrm{~cm}^{-1}\right)$ : $1596,1627,2997,3050 ;{ }^{1} \mathrm{H}-\mathrm{NMR}\left(400 \mathrm{MHz}\right.$, DMSO- $d_{6}, \delta$ ppm) $1.72-1.91$ $\left(\mathrm{m}, 3 \mathrm{H}, \mathrm{CH}_{2}\right), 2.12-2.22\left(\mathrm{~m}, 1 \mathrm{H}, \mathrm{CH}_{2}\right), 3.48-3.56\left(\mathrm{~m}, 1 \mathrm{H}, \mathrm{CH}_{2}\right), 3.72-3.80\left(\mathrm{~m}, 1 \mathrm{H}, \mathrm{CH}_{2}\right), 5.14-5.20(\mathrm{~m}$, $1 \mathrm{H}, \mathrm{CH}), 5.85\left(\mathrm{~s}, 2 \mathrm{H}, \mathrm{CH}_{2}\right), 6.44(\mathrm{~s}, 1 \mathrm{H}, \mathrm{Ar}-\mathrm{H}), 6.52(\mathrm{~s}, 1 \mathrm{H}, \mathrm{Ar}-\mathrm{H}), 6.90(\mathrm{t}, 1 \mathrm{H}, J=7.4 \mathrm{~Hz}, \mathrm{Ar}-\mathrm{H}), 7.20(\mathrm{t}$, $2 \mathrm{H}, J=7.8 \mathrm{~Hz}, \mathrm{Ar}-\mathrm{H}), 7.43(\mathrm{~d}, 2 \mathrm{H}, J=8.1 \mathrm{~Hz}, \mathrm{Ar}-\mathrm{H}), 7.97(\mathrm{~s}, 1 \mathrm{H}, \mathrm{NH}), 9.33(\mathrm{~s}, 1 \mathrm{H}, \mathrm{OH}) ;{ }^{13} \mathrm{C}-\mathrm{NMR}(151$ MHz, DMSO- $d_{6}, \delta$ ppm) 23.7, 33.3, 47.1, 55.7, 98.2, 101.0, 106.1, 119.8, 122.1, 122.7, 128.7, 140.2, 140.9, 146.4, 148.8, 154.1; Elemental analysis: calc. for $\mathrm{C}_{18} \mathrm{H}_{18} \mathrm{~N}_{2} \mathrm{O}_{4}$ (326): $\mathrm{C}, 66.25 ; \mathrm{H}, 5.56 ; \mathrm{N}, 8.58$; found $\mathrm{C}$, 66.31; $\mathrm{H}, 5.70 ; \mathrm{N}, 8.35$; ESI $m / z:[\mathrm{M}+\mathrm{H}]^{+}$: calc. for $\mathrm{C}_{18} \mathrm{H}_{19} \mathrm{~N}_{2} \mathrm{O}_{4} 327$; found 327 .

2-(6-Hydroxybenzo[d][1,3]dioxol-5-yl)-N-(4-methoxyphenyl)pyrrolidine-1-carboxamide (5c). Beige solid, yield $91 \%$, m.p. $112-114{ }^{\circ} \mathrm{C}$; IR $\left(v, \mathrm{~cm}^{-1}\right)$ : $1597,1627,2971,2989,3037 ;{ }^{1} \mathrm{H}-\mathrm{NMR}$ (400 MHz, DMSO- $d_{6}$, $\delta$ ppm) 1.72-1.92 (m, 3H, $\left.\mathrm{CH}_{2}\right), 2.10-2.27\left(\mathrm{~m}, 1 \mathrm{H}, \mathrm{CH}_{2}\right), 3.45-3.56\left(\mathrm{~m}, 2 \mathrm{H}, \mathrm{CH}_{2}\right), 3.68\left(\mathrm{~s}, 3 \mathrm{H}, \mathrm{CH}_{3}\right)$, 5.12-5.22 (m, 1H, CH), $5.85\left(\mathrm{~s}, 2 \mathrm{H}, \mathrm{CH}_{2}\right), 6.46(\mathrm{~s}, 1 \mathrm{H}, \mathrm{Ar}-\mathrm{H}), 6.52(\mathrm{~s}, 1 \mathrm{H}, \mathrm{Ar}-\mathrm{H}), 6.79(\mathrm{~d}, 2 \mathrm{H}, J=9.1 \mathrm{~Hz}$, Ar-H), 7.33 (d, 2H, J = 9.0 Hz, Ar-H), $7.85(\mathrm{~s}, 1 \mathrm{H}, \mathrm{NH}), 9.39$ (s, $1 \mathrm{H}, \mathrm{OH}) ;{ }^{13} \mathrm{C}-\mathrm{NMR}\left(151 \mathrm{MHz}, \mathrm{DMSO}-d_{6}\right.$, $\delta$ ppm) 23.7, 33.2, 47.0, 55.6, 55.6, 98.3, 100.9, 106.2, 114.0, 121.8, 128. 8, 133.9, 140.2, 146.4, 148.9, 154.5, 154.9; Elemental analysis: calc. for $\mathrm{C}_{19} \mathrm{H}_{20} \mathrm{~N}_{2} \mathrm{O}_{5}$ (356): $\mathrm{C}, 64.04 ; \mathrm{H}, 5.66 ; \mathrm{N}, 7.86$; found $\mathrm{C}, 64.21 ; \mathrm{H}, 5.87$; $\mathrm{N}, 7.94 ; \mathrm{ESI} m / z:[\mathrm{M}+\mathrm{H}]^{+}$: calc. for $\mathrm{C}_{19} \mathrm{H}_{21} \mathrm{~N}_{2} \mathrm{O}_{5} 357$; found 357 .

N-(4-Bromophenyl)-2-(6-hydroxybenzo[d][1,3]dioxol-5-yl)pyrrolidine-1-carboxamide (5d). White solid, yield $95 \%$, m.p. $164{ }^{\circ} \mathrm{C}$; IR $\left(v, \mathrm{~cm}^{-1}\right)$ : $1595,1627,2848,2978,3047 ;{ }^{1} \mathrm{H}-\mathrm{NMR}\left(400 \mathrm{MHz}\right.$, DMSO- $d_{6}, \delta$ ppm) 1.71-1.94 (m, 3H, CH $), 2.11-2.23\left(\mathrm{~m}, 1 \mathrm{H}, \mathrm{CH}_{2}\right), 3.46-3.57\left(\mathrm{~m}, 1 \mathrm{H}, \mathrm{CH}_{2}\right), 3.70-3.81\left(\mathrm{~m}, 1 \mathrm{H}, \mathrm{CH}_{2}\right)$, 5.14-5.24 (m, 1H, CH), $5.85\left(\mathrm{~s}, 2 \mathrm{H}, \mathrm{CH}_{2}\right), 6.44(\mathrm{~s}, 1 \mathrm{H}, \mathrm{Ar}-\mathrm{H}), 6.49(\mathrm{~s}, 1 \mathrm{H}, \mathrm{Ar}-\mathrm{H}), 7.37(\mathrm{~d}, 2 \mathrm{H}, J=8.8 \mathrm{~Hz}$, Ar-H), $7.44(\mathrm{~d}, 2 \mathrm{H}, J=8.7 \mathrm{~Hz}, \mathrm{Ar}-\mathrm{H}), 8.17(\mathrm{~s}, 1 \mathrm{H}, \mathrm{NH}), 9.30(\mathrm{~s}, 1 \mathrm{H}, \mathrm{OH}) ;{ }^{13} \mathrm{C}-\mathrm{NMR}\left(151 \mathrm{MHz}\right.$, DMSO-d $d_{6}$, $\delta$ ppm) 23.7, 33.2, 47.1, 55.9, 98.2, 100.9, 106.1, 113.5, 121.6, 122.6, 131.5, 140.1, 140.4, 146.3, 148.8, 153.9; Elemental analysis: calc. for $\mathrm{C}_{18} \mathrm{H}_{17} \mathrm{BrN}_{2} \mathrm{O}_{4}$ (405): $\mathrm{C}, 53.35 ; \mathrm{H}, 4.23 ; \mathrm{Br}, 19.72 ; \mathrm{N}, 6.91$; found $\mathrm{C}, 53.41$; $\mathrm{H}, 4.33 ; \mathrm{Br}, 19.79 ; \mathrm{N}, 6.79$; $\mathrm{ESI} m / z:[\mathrm{M}+\mathrm{H}]^{+}$: calc. for $\mathrm{C}_{12} \mathrm{H}_{8} \mathrm{ClN}_{4} \mathrm{O}_{5} 323$; found 323; $\mathrm{ESI} m / z:[\mathrm{M}+\mathrm{H}]^{+}$: calc. for $\mathrm{C}_{18} \mathrm{H}_{18} \mathrm{BrN}_{2} \mathrm{O}_{4} 406$; found 406 .

$\mathrm{N}$-(4-Fluorophenyl)-2-(6-hydroxybenzo[d][1,3]dioxol-5-yl)pyrrolidine-1-carboxamide (5e). White solid, yield $91 \%$, m.p. $184-185^{\circ} \mathrm{C}$; IR $\left(v, \mathrm{~cm}^{-1}\right)$ : $1595,1638,2883,2948,2989,3164 ;{ }^{1} \mathrm{H}-\mathrm{NMR}(400 \mathrm{MHz}$, DMSO- $\left.d_{6}, \delta \mathrm{ppm}\right) 1.72-1.83\left(\mathrm{~m}, 2 \mathrm{H}, \mathrm{CH}_{2}\right), 1.88-1.98\left(\mathrm{~m}, 1 \mathrm{H}, \mathrm{CH}_{2}\right), 2.11-2.28\left(\mathrm{~m}, 1 \mathrm{H}, \mathrm{CH}_{2}\right), 3.48-3.59$ $\left(\mathrm{m}, 1 \mathrm{H}, \mathrm{CH}_{2}\right), 3.68-3.78\left(\mathrm{~m}, 1 \mathrm{H}, \mathrm{CH}_{2}\right), 5.17-5.27(\mathrm{~m}, 1 \mathrm{H}, \mathrm{CH}), 6.57\left(\mathrm{~d}, 2 \mathrm{H}, J=7.9 \mathrm{~Hz}, \mathrm{CH}_{2}\right), 6.64(\mathrm{~s}$, $1 \mathrm{H}, \mathrm{Ar}-\mathrm{H}), 6.85\left(\mathrm{~d}, 1 \mathrm{H}, J=8.2 \mathrm{~Hz}, \mathrm{CH}_{2}\right), 6.99-7.07(\mathrm{~m}, 2 \mathrm{H}, \mathrm{Ar}-\mathrm{H}), 7.33(\mathrm{~s}, 1 \mathrm{H}, \mathrm{Ar}-\mathrm{H}), 7.40-7.48(\mathrm{~m}, 2 \mathrm{H}$, Ar-H), 8.09 (s, 1H, Ar-H), 9.72 (s, 1H,NH), $9.84(\mathrm{~s}, 1 \mathrm{H}, \mathrm{OH}) ;{ }^{13} \mathrm{C}-\mathrm{NMR}\left(151 \mathrm{MHz}, \mathrm{DMSO}-d_{6}, \delta \mathrm{ppm}\right) 23.6$, 32.8, 47.1, 55.9, 102.2, 110.2, 113.7, $115.1(\mathrm{~d}, J=22.0 \mathrm{~Hz}), 121.6(\mathrm{~d}, J=7.6 \mathrm{~Hz}), 126.1,130.5,133.2,137.8$ $(\mathrm{d}, J=124.5 \mathrm{~Hz}), 148.3,154.2,157.5(\mathrm{~d}, J=137.9 \mathrm{~Hz})$; Elemental analysis: calc. for $\mathrm{C}_{18} \mathrm{H}_{17} \mathrm{FN}_{2} \mathrm{O}_{4}(344)$ : C, 62.79; $\mathrm{H}, 4.98 ; \mathrm{N}, 8.14$; found $\mathrm{C}, 62.93 ; \mathrm{H}, 5.09 ; \mathrm{N}, 8.30 ; \mathrm{ESI} \mathrm{m} / z$ : $[\mathrm{M}+\mathrm{H}]^{+}$: calc. for $\mathrm{C}_{18} \mathrm{H}_{18} \mathrm{FN}_{2} \mathrm{O}_{4}$ 345 ; found 345 .

N-Hexyl-2-(6-hydroxybenzo[d][1,3]dioxol-5-yl)pyrrolidine-1-carboxamide (5f). White solid, yield 72\%, m.p. $91-93{ }^{\circ} \mathrm{C}$; IR $\left(v, \mathrm{~cm}^{-1}\right)$ : 1535, 1624, 2720, 2855, 2929, 3115; ${ }^{1} \mathrm{H}-\mathrm{NMR}\left(400 \mathrm{MHz}, \mathrm{CDCl}_{3}, \delta \mathrm{ppm}\right)$ $0.87\left(\mathrm{t}, 3 \mathrm{H}, J=6.9 \mathrm{~Hz}, \mathrm{CH}_{3}\right), 1.23-1.31\left(\mathrm{~m}, 6 \mathrm{H}, \mathrm{CH}_{2}\right), 1.42-1.51\left(\mathrm{~m}, 2 \mathrm{H}, \mathrm{CH}_{2}\right), 2.04-2.13\left(\mathrm{~m}, 2 \mathrm{H}, \mathrm{CH}_{2}\right)$, 2.18-2.32 (m, 2H, $\left.\mathrm{CH}_{2}\right), 3.10-3.16\left(\mathrm{~m}, 1 \mathrm{H}, \mathrm{CH}_{2}\right), 3.19-3.28\left(\mathrm{~m}, 1 \mathrm{H}, \mathrm{CH}_{2}\right), 3.40-3.54\left(\mathrm{~m}, 2 \mathrm{H}, \mathrm{CH}_{2}\right)$, 5.12-5.21 (m, 1H, CH), $5.85\left(\mathrm{~d}, 2 \mathrm{H}, J=12.4 \mathrm{~Hz}, \mathrm{CH}_{2}\right), 6.47(\mathrm{~s}, 1 \mathrm{H}, \mathrm{Ar}-\mathrm{H}), 6.61(\mathrm{~s}, 1 \mathrm{H}, \mathrm{Ar}-\mathrm{H}) ;{ }^{13} \mathrm{C}-\mathrm{NMR}$ (151 MHz, $\mathrm{CDCl}_{3}, \delta$ ppm) 14.0, 22.6, 24.9, 26.5, 30.1, 31.5, 32.7, 40.9, 46.4, 55.0, 99.9, 100.9, 105.3, 120.8, 141.0, 147.3, 150.4, 158.0; Elemental analysis: calc. for $\mathrm{C}_{18} \mathrm{H}_{26} \mathrm{~N}_{2} \mathrm{O}_{4}$ (334): C, 64.65; H, 7.84; N, 8.38; found $\mathrm{C}, 64.75 ; \mathrm{H}, 8.06 ; \mathrm{N}, 8.25 ; \mathrm{ESI} m / z$ : $[\mathrm{M}+\mathrm{H}]^{+}$: calc. for $\mathrm{C}_{18} \mathrm{H}_{27} \mathrm{~N}_{2} \mathrm{O}_{4} 335$; found 335 .

N-Cyclohexyl-2-(6-hydroxybenzo[d][1,3]dioxol-5-yl)pyrrolidine-1-carboxamide (5g). White solid, yield 84\%, m.p. ${ }^{159-160}{ }^{\circ} \mathrm{C}$; IR $\left(v, \mathrm{~cm}^{-1}\right)$ : 1528, 1624, 2720, 2855, 2929, 3113, 3403; ${ }^{1} \mathrm{H}-\mathrm{NMR}(400 \mathrm{MHz}$, 
DMSO- $\left.d_{6}, \delta \mathrm{ppm}\right) 1.03-1.12\left(\mathrm{~m}, 2 \mathrm{H}, \mathrm{CH}_{2}\right), 1.13-1.25\left(\mathrm{~m}, 3 \mathrm{H}, \mathrm{CH}_{2}\right), 1.47-1.66\left(\mathrm{~m}, 4 \mathrm{H}, \mathrm{CH}_{2}\right), 1.69-1.78$ $\left(\mathrm{m}, 2 \mathrm{H}, \mathrm{CH}_{2}\right), 1.79-1.90\left(\mathrm{~m}, 2 \mathrm{H}, \mathrm{CH}_{2}\right), 2.07-2.19\left(\mathrm{~m}, 1 \mathrm{H}, \mathrm{CH}_{2}\right), 3.31-3.43\left(\mathrm{~m}, 2 \mathrm{H}, \mathrm{CH}_{2}\right), 3.47-3.54(\mathrm{~m}$, $\left.1 \mathrm{H}, \mathrm{CH}_{2}\right), 4.95-5.04(\mathrm{~m}, 1 \mathrm{H}, \mathrm{CH}), 5.85\left(\mathrm{~d}, 2 \mathrm{H}, J=8.2 \mathrm{~Hz}, \mathrm{CH}_{2}\right), 6.42(\mathrm{~s}, 1 \mathrm{H}, \mathrm{Ar}-\mathrm{H}), 6.47(\mathrm{~s}, 1 \mathrm{H}, \mathrm{Ar}-\mathrm{H})$; ${ }^{13} \mathrm{C}-\mathrm{NMR}\left(151 \mathrm{MHz}, \mathrm{DMSO}-d_{6}, \delta\right.$ ppm) 23.9, 25.2, 25.8, 33.5, 33.6, 46.7, 49.1, 55.0, 98.3, 100.9, 106.2, 122.7, 140.3, 146.5, 149.1, 156.3; Elemental analysis: calc. for $\mathrm{C}_{18} \mathrm{H}_{24} \mathrm{~N}_{2} \mathrm{O}_{4}$ (332): C, 65.04; $\mathrm{H}, 7.28$; $\mathrm{N}$, 8.43; found $\mathrm{C}, 65.16 ; \mathrm{H}, 7.42 ; \mathrm{N}, 8.35$; ESI $m / z$ : $[\mathrm{M}+\mathrm{H}]^{+}$: calc. for $\mathrm{C}_{18} \mathrm{H}_{25} \mathrm{~N}_{2} \mathrm{O}_{4} 333$; found 333 .

2-(2-(6-Hydroxybenzo[d][1,3]dioxol-5-yl)pyrrolidine-1-carboxamido)- $N, N$-dimethylethan-1-aminium 2,2,2-trifluoroacetate (5h). White solid, yield $75 \%$, m.p. $171-172{ }^{\circ} \mathrm{C}$; $\mathrm{IR}\left(v, \mathrm{~cm}^{-1}\right)$ : 1527, 1626, 2838, 2929, 3113,$3394 ;{ }^{1} \mathrm{H}-\mathrm{NMR}\left(400 \mathrm{MHz}\right.$, DMSO- $\left.d_{6}, \delta \mathrm{ppm}\right) 1.69-1.80\left(\mathrm{~m}, 2 \mathrm{H}, \mathrm{CH}_{2}\right), 1.81-1.89\left(\mathrm{~m}, 1 \mathrm{H}, \mathrm{CH}_{2}\right)$, 2.04-2.09 (m, $\left.1 \mathrm{H}, \mathrm{CH}_{2}\right), 2.79\left(\mathrm{~s}, 6 \mathrm{H}, \mathrm{CH}_{3}\right), 3.06-3.16\left(\mathrm{~m}, 2 \mathrm{H}, \mathrm{CH}_{2}\right), 3.31-3.37\left(\mathrm{~m}, 3 \mathrm{H}, \mathrm{CH}_{2}\right), 3.53-3.60(\mathrm{~m}$, $\left.1 \mathrm{H}, \mathrm{CH}_{2}\right), 4.99-5.05(\mathrm{~m}, 1 \mathrm{H}, \mathrm{CH}), 5.85\left(\mathrm{~d}, 2 \mathrm{H}, J=11.0 \mathrm{~Hz}, \mathrm{CH}_{2}\right), 6.42(\mathrm{~s}, 1 \mathrm{H}, \mathrm{Ar}-\mathrm{H}), 6.44(\mathrm{~s}, 1 \mathrm{H}, \mathrm{Ar}-\mathrm{H})$, 9.37 (s, $1 \mathrm{H}, \mathrm{NH}), 9.63(\mathrm{~s}, 1 \mathrm{H}, \mathrm{OH}) ;{ }^{13} \mathrm{C}-\mathrm{NMR}\left(151 \mathrm{MHz}, \mathrm{DMSO}-d_{6}, \delta \mathrm{ppm}\right) 23.4,33.2,35.9,43.1,46.7$, $55.9,57.9,98.3,100.9,106.1,117.7(\mathrm{q}, J=300.4 \mathrm{~Hz}), 122.8,139.9,146.2,148.9,157.1,158.6(\mathrm{q}, J=31.0 \mathrm{~Hz})$; Elemental analysis: calc. for $\mathrm{C}_{18} \mathrm{H}_{24} \mathrm{~F}_{3} \mathrm{~N}_{3} \mathrm{O}_{6}$ (435): $\mathrm{C}, 49.66 ; \mathrm{H}, 5.56 ; \mathrm{N}, 9.65$; found $\mathrm{C}, 49.85 ; \mathrm{H}, 5.67 ; \mathrm{N}$, 9.80; ESI $m / z$ : $\left[\mathrm{M}-\mathrm{CF}_{3} \mathrm{CO}_{2}\right]^{+}$: calc. for $\mathrm{C}_{16} \mathrm{H}_{24} \mathrm{~N}_{3} \mathrm{O}_{4} 322$; found 322 .

6-Chloro-4-((3-hydroxy-4-(1-(phenylcarbamoyl)pyrrolidin-2-yl)phenyl)amino)-5-nitrobenzo[c][1,2,5]oxadia zole 1-oxide (6b). Dark solid, yield 87\%, m.p. $165-170{ }^{\circ} \mathrm{C}$ with decomposition; IR $\left(v, \mathrm{~cm}^{-1}\right)$ : 753, 1383, 1559, 1627, 3073, 3336, 3396; ${ }^{1} \mathrm{H}-\mathrm{NMR}\left(400 \mathrm{MHz}, \mathrm{DMSO}-d_{6}, \delta \mathrm{ppm}\right) 1.80\left(\mathrm{~m}, 2 \mathrm{H}, \mathrm{CH}_{2}\right), 1.91(\mathrm{~m}, 1 \mathrm{H}$, $\left.\mathrm{CH}_{2}\right), 2.20\left(\mathrm{~m}, 1 \mathrm{H}, \mathrm{CH}_{2}\right), 3.53-3.54\left(\mathrm{~m}, 1 \mathrm{H}, \mathrm{CH}_{2}\right), 3.75\left(\mathrm{~m}, 1 \mathrm{H}, \mathrm{CH}_{2}\right), 5.22-5.23(\mathrm{~m}, 1 \mathrm{H}, \mathrm{CH}), 6.57(\mathrm{~d}, 1 \mathrm{H}$, $J=7.7 \mathrm{~Hz}, \mathrm{Ar}-\mathrm{H}), 6.64(\mathrm{~s}, 1 \mathrm{H}, \mathrm{Ar}-\mathrm{H}), 6.87(\mathrm{~d}, 1 \mathrm{H}, J=8.2 \mathrm{~Hz}, \mathrm{Ar}-\mathrm{H}), 6.91(\mathrm{t}, 1 \mathrm{H}, J=7.40 \mathrm{~Hz}, \mathrm{Ar}-\mathrm{H}), 7.20$ $(\mathrm{t}, 2 \mathrm{H}, J=7.9 \mathrm{~Hz}, \mathrm{Ar}-\mathrm{H}), 7.33(\mathrm{~s}, 1 \mathrm{H}, \mathrm{Ar}-\mathrm{H}), 7.43(\mathrm{~d}, 2 \mathrm{H}, J=7.1 \mathrm{~Hz}, \mathrm{Ar}-\mathrm{H}), 7.99$ (s, 1H, NH), $9.74(\mathrm{~s}, 1 \mathrm{H}$, $\mathrm{NH}), 9.84(\mathrm{~s}, 1 \mathrm{H}, \mathrm{OH}) ;{ }^{13} \mathrm{C}-\mathrm{NMR}\left(151 \mathrm{MHz}, \mathrm{DMSO}_{6}, \delta \mathrm{ppm}\right) 23.7,33.0,47.1,55.8,102.3,110.3,113.9$, 114.3, 119.9, 122.1, 126.2, 127.1, 128.1, 128.7, 130.6, 133.3, 138.5, 140.9, 148.3, 154.2, 154.4; Elemental analysis: calc. for $\mathrm{C}_{23} \mathrm{H}_{19} \mathrm{ClN}_{6} \mathrm{O}_{6}$ (510.9): $\mathrm{C} 54.07 ; \mathrm{H} 3.75 ; \mathrm{Cl} 6.94 ; \mathrm{N} 16.45$; found $\mathrm{C} 54.18 ; \mathrm{H} 3.83 ; \mathrm{Cl}$ 6.85; $\mathrm{N}$ 16.32; ESI $m / z:[\mathrm{M}+\mathrm{H}]^{+}$: calc. for $\mathrm{C}_{23} \mathrm{H}_{20} \mathrm{ClN}_{6} \mathrm{O}_{6} 511.9$; found 512 .

6-Chloro-4-((3-hydroxy-4-(1-((4-methoxyphenyl)carbamoyl)pyrrolidin-2-yl)phenyl)amino)-5-nitrobenzo[c] [1,2,5]oxadiazole 1-oxide (6c). Dark solid, yield 89\%, m.p. $196-202{ }^{\circ} \mathrm{C}$ with decomposition; IR $\left(\mathrm{v}, \mathrm{cm}^{-1}\right)$ : 750, 1377, 1557, 1628, 3075, 3306, 3391; ${ }^{1} \mathrm{H}-\mathrm{NMR}\left(400 \mathrm{MHz}\right.$, DMSO- $\left.d_{6}, \delta \mathrm{ppm}\right) 1.73-1.84\left(\mathrm{~m}, 2 \mathrm{H}, \mathrm{CH}_{2}\right)$, 1.86-1.94 (m, 1H, $\left.\mathrm{CH}_{2}\right), 2.12-2.23\left(\mathrm{~m}, 1 \mathrm{H}, \mathrm{CH}_{2}\right), 3.47-3.53\left(\mathrm{~m}, 1 \mathrm{H}, \mathrm{CH}_{2}\right), 3.69\left(\mathrm{~s}, 3 \mathrm{H}, \mathrm{CH}_{3}\right), 3.69-3.74(\mathrm{~m}$, $\left.1 \mathrm{H}, \mathrm{CH}_{2}\right), 5.16-5.23(\mathrm{~m}, 1 \mathrm{H}, \mathrm{CH}), 6.57(\mathrm{~d}, 1 \mathrm{H}, J=7.6 \mathrm{~Hz}, \mathrm{Ar}-\mathrm{H}), 6.63(\mathrm{~s}, 1 \mathrm{H}, \mathrm{Ar}-\mathrm{H}), 6.78(\mathrm{~d}, 2 \mathrm{H}, J=9.02$ $\mathrm{Hz}, \mathrm{Ar}-\mathrm{H}), 6.87(\mathrm{~d}, 1 \mathrm{H}, J=8.3 \mathrm{~Hz}, \mathrm{Ar}-\mathrm{H}), 7.31(\mathrm{~s}, 1 \mathrm{H}, \mathrm{Ar}-\mathrm{H}), 7.33(\mathrm{~d}, 2 \mathrm{H}, J=4.8 \mathrm{~Hz}, \mathrm{Ar}-\mathrm{H}), 7.86$ (br s, 1H, NH), 9.73 (br s, 1H, NH), 9.83 (nr s, $1 \mathrm{H}, \mathrm{OH}) ;{ }^{13} \mathrm{C}-\mathrm{NMR}$ (151 MHz, DMSO-d 6 , $\delta$ ppm) 23.7, 32.1, 32.9, 47.1, 55.6, 102.2, 107.0, 110.3, 114.0, 121.8, 121.9, 123.0, 124.5, 127.1, 130.6, 133.9, 138.5, 141.3, 146.7, 148.2, 154.5, 154.9; Elemental analysis: calc. for $\mathrm{C}_{24} \mathrm{H}_{21} \mathrm{ClN}_{6} \mathrm{O}_{7}$ (540.9): $\mathrm{C} 53.29 ; \mathrm{H} 3.91 ; \mathrm{Cl} 6.55 ; \mathrm{N}$ 15.54; found C 53.38; $\mathrm{H} 3.81 ; \mathrm{Cl}$ 6.42; N 15.67; ESI $m / z$ : $[\mathrm{M}+\mathrm{H}]^{+}$: calc. for $\mathrm{C}_{24} \mathrm{H}_{22} \mathrm{ClN}_{6} \mathrm{O}_{7} 541.9$; found 542 .

4-((4-(1-((4-Bromophenyl)carbamoyl)pyrrolidin-2-yl)-3-hydroxyphenyl)amino)-6-chloro-5-nitrobenzo[c] [1,2,5]oxadiazole 1-oxide (6d). Dark solid, yield 95\%, m.p. $166-170{ }^{\circ} \mathrm{C}$ with decomposition; IR $\left(v, \mathrm{~cm}^{-1}\right)$ : 753, 1362, 1560, 1629, 3100, 3297, 3382; ${ }^{1} \mathrm{H}-\mathrm{NMR}\left(400 \mathrm{MHz}\right.$, DMSO- $\left.d_{6}, \delta \mathrm{ppm}\right) 1.74-1.84\left(\mathrm{~m}, 2 \mathrm{H}, \mathrm{CH}_{2}\right)$, 1.84-1.97 (m, $\left.1 \mathrm{H}, \mathrm{CH}_{2}\right), 2.15-2.24\left(\mathrm{~m}, 1 \mathrm{H}, \mathrm{CH}_{2}\right), 3.47-3.57\left(\mathrm{~m}, 1 \mathrm{H}, \mathrm{CH}_{2}\right), 3.68-3.77\left(\mathrm{~m}, 1 \mathrm{H}, \mathrm{CH}_{2}\right)$, 5.18-5.25 (m, 1H, CH), $6.56(\mathrm{~d}, 1 \mathrm{H}, J=8.2 \mathrm{~Hz}, \mathrm{Ar}-\mathrm{H}), 6.63(\mathrm{~s}, 1 \mathrm{H}, \mathrm{Ar}-\mathrm{H}), 6.85(\mathrm{~d}, 1 \mathrm{H}, J=8.24 \mathrm{~Hz}, \mathrm{Ar}-\mathrm{H})$, $7.33(\mathrm{~s}, 1 \mathrm{H}, \mathrm{Ar}-\mathrm{H}), 7.36$ (d, 2H, J = 8.8 Hz, Ar-H), $7.44(\mathrm{t}, 2 \mathrm{H}, J=8.8 \mathrm{~Hz}, \mathrm{Ar}-\mathrm{H}), 8.19$ (br s, 1H, NH), 9.71 (br s, $1 \mathrm{H}, \mathrm{NH}), 9.83$ (br s, $1 \mathrm{H}, \mathrm{OH}) ;{ }^{13} \mathrm{C}-\mathrm{NMR}\left(151 \mathrm{MHz}, \mathrm{DMSO}-d_{6}, \delta \mathrm{ppm}\right) 23.6,32.9,47.1,56.0,110.2$, 113.5, 113.8, 114.3, 120.8, 121.7, 126.1, 127.1, 130.6, 131.4, 132.0, 138.5, 139.5, 140.4, 148.3, 153.9, 154.4; Elemental analysis: calc. for $\mathrm{C}_{23} \mathrm{H}_{18} \mathrm{BrClN}_{6} \mathrm{O}_{6}$ (589.8): $\mathrm{C} 46.84 ; \mathrm{H} 3.08 ; \mathrm{Cl} 6.01 ; \mathrm{N} 14.25$; found $\mathrm{C} 46.92$; $\mathrm{H} 3.12 ; \mathrm{Cl} 6.05 ; \mathrm{N} 14.34 ; \mathrm{ESI} m / z$ : $[\mathrm{M}+\mathrm{H}]^{+}$: calc. for $\mathrm{C}_{23} \mathrm{H}_{19} \mathrm{BrClN}_{6} \mathrm{O}_{6} 590.8$; found 591 .

6-Chloro-4-((4-(1-((4-fluorophenyl)carbamoyl)pyrrolidin-2-yl)-3-hydroxyphenyl)amino)-5-nitrobenzo[c] [1,2,5]oxadiazole 1-oxide (6e). Dark solid, yield $76 \%$, m.p. $175-177^{\circ} \mathrm{C}$ with decomposition; IR $\left(\nu, \mathrm{cm}^{-1}\right)$ : $1348,1564,1623,3247,3404 ;{ }^{1} \mathrm{H}-\mathrm{NMR}\left(400 \mathrm{MHz}, \mathrm{DMSO}-d_{6}, \delta \mathrm{ppm}\right) 1.65-1.83\left(\mathrm{~m}, 2 \mathrm{H}, \mathrm{CH}_{2}\right), 1.86-1.98$ $\left(\mathrm{m}, 1 \mathrm{H}, \mathrm{CH}_{2}\right), 2.11-2.27\left(\mathrm{~m}, 1 \mathrm{H}, \mathrm{CH}_{2}\right), 3.49-3.58\left(\mathrm{~m}, 1 \mathrm{H}, \mathrm{CH}_{2}\right), 3.69-3.79\left(\mathrm{~m}, 1 \mathrm{H}, \mathrm{CH}_{2}\right), 5.16-5.25(\mathrm{~m}$, $1 \mathrm{H}, \mathrm{CH}), 6.57(\mathrm{dd}, 1 \mathrm{H}, J=8.2 \mathrm{~Hz}, J=2.1 \mathrm{~Hz}, \mathrm{Ar}-\mathrm{H}), 6.64(\mathrm{~d}, 1 \mathrm{H}, J=2.1 \mathrm{~Hz}, \mathrm{Ar}-\mathrm{H}), 6.85(\mathrm{~d}, 1 \mathrm{H}, J=8.1$ 
Hz, Ar-H), 6.98-7.07 (m, 2H, Ar-H), 7.33 (s, 1H, Ar-H), 7.41-7.49 (m, 2H, Ar-H), 8.09 (s, 1H, NH), 9.72 $(\mathrm{s}, 1 \mathrm{H}, \mathrm{NH}), 9.84(\mathrm{~s}, 1 \mathrm{H}, \mathrm{OH}) ;{ }^{13} \mathrm{C}-\mathrm{NMR}\left(151 \mathrm{MHz}, \mathrm{DMSO}-d_{6}, \delta \mathrm{ppm}\right) 23.6,32.8,47.1,55.9,102.2,110.2$, $113.7,114.2,115.10(\mathrm{~d}, J=22.0 \mathrm{~Hz}), 121.6(\mathrm{~d}, J=7.6 \mathrm{~Hz}), 126.1,127.1,128.0,130.5,133.2,137.2(\mathrm{~d}, J=2.1$ $\mathrm{Hz}), 138.4,148.3,154.2,157.7(\mathrm{~d}, J=237.9 \mathrm{~Hz})$; Elemental analysis: calc. for $\mathrm{C}_{23} \mathrm{H}_{18} \mathrm{ClFN}_{6} \mathrm{O}_{6}$ (528.9): $\mathrm{C}$, $52.23 ; \mathrm{H}, 3.43 ; \mathrm{Cl}, 6.70 ; \mathrm{N}, 15.89$; found $\mathrm{C}, 52.00 ; \mathrm{H}, 3.61 ; \mathrm{Cl}, 6.87 ; \mathrm{N}, 16.07 ; \mathrm{ESI} m / z:[\mathrm{M}+\mathrm{H}]^{+}$: calc. for $\mathrm{C}_{23} \mathrm{H}_{19} \mathrm{ClFN}_{6} \mathrm{O}_{6} 529.9$; found 530 .

6-Chloro-4-((4-(1-(hexylcarbamoyl)pyrrolidin-2-yl)-3-hydroxyphenyl)amino)-5-nitrobenzo[c][1,2,5]oxadia zole 1-oxide (6f). Dark solid, yield 44\%, m.p. $121-122{ }^{\circ} \mathrm{C}$ with decomposition; IR $\left(v, \mathrm{~cm}^{-1}\right)$ : 722, 1347, 1563, 1630, 3106, 3194, 3426; ${ }^{1} \mathrm{H}-\mathrm{NMR}\left(400 \mathrm{MHz}, \mathrm{DMSO}-d_{6}, \delta \mathrm{ppm}\right) 0.84\left(\mathrm{t}, 3 \mathrm{H}, J=6.7 \mathrm{~Hz}, \mathrm{CH}_{3}\right)$, $1.17-1.26\left(\mathrm{~m}, 6 \mathrm{H}, \mathrm{CH}_{2}\right), 1.31-1.38\left(\mathrm{~m}, 2 \mathrm{H}, \mathrm{CH}_{2}\right), 1.71-1.79\left(\mathrm{~m}, 2 \mathrm{H}, \mathrm{CH}_{2}\right), 1.80-1.90\left(\mathrm{~m}, 1 \mathrm{H}, \mathrm{CH}_{2}\right)$, 2.07-2.18 (m, 1H, $\left.\mathrm{CH}_{2}\right), 2.90-3.02\left(\mathrm{~m}, 2 \mathrm{H}, \mathrm{CH}_{2}\right), 3.42-3.54\left(\mathrm{~m}, 2 \mathrm{H}, \mathrm{CH}_{2}\right), 4.99-5.06(\mathrm{~m}, 1 \mathrm{H}, \mathrm{CH}), 5.85$ (br s, 1H, NH), $6.55(\mathrm{~d}, 1 \mathrm{H}, J=7.2 \mathrm{~Hz}, \mathrm{Ar}-\mathrm{H}), 6.61(\mathrm{~s}, 1 \mathrm{H}, \mathrm{Ar}-\mathrm{H}), 6.79(\mathrm{~d}, 1 \mathrm{H}, J=8.3 \mathrm{~Hz}, \mathrm{Ar}-\mathrm{H}), 7.34(\mathrm{~s}, 1 \mathrm{H}$, Ar-H), 9.01 (br s, 1H, NH), 9.85 (br s, $1 \mathrm{H}, \mathrm{OH}) ;{ }^{13} \mathrm{C}-\mathrm{NMR}\left(151 \mathrm{MHz}, \mathrm{DMSO}-d_{6}, \delta\right.$ ppm) 14.4, 22.5, 23.7, 26.5, 30.4, 31.5, 32.9, 46.7, 55.3, 102.3, 110.4, 113.7, 126.3, 127.1, 128.3, 130.6, 133.3, 134.9, 138.5, 148.3, 154.6, 157.0; Elemental analysis: calc. for $\mathrm{C}_{23} \mathrm{H}_{27} \mathrm{ClN}_{6} \mathrm{O}_{6}$ (518.9): $\mathrm{C} 53.23 ; \mathrm{H}$ 5.24; $\mathrm{Cl}$ 6.83; $\mathrm{N}$ 16.19; found $\mathrm{C} 53.35 ; \mathrm{H} 5.33 ; \mathrm{Cl} 6.72 ; \mathrm{N} 16.12 ; \mathrm{ESI} m / z$ : $[\mathrm{M}+\mathrm{H}]^{+}$: calc. for $\mathrm{C}_{23} \mathrm{H}_{28} \mathrm{ClN}_{6} \mathrm{O}_{6} 519.9$; found 520 .

6-Chloro-4-((4-(1-(cyclohexylcarbamoyl)pyrrolidin-2-yl)-3-hydroxyphenyl)amino)-5-nitrobenzo[c][1,2,5] oxadiazole 1-oxide (6g). Dark solid, yield $67 \%$, m.p. $170-175^{\circ} \mathrm{C}$ with decomposition; IR $\left(\mathrm{v}, \mathrm{cm}^{-1}\right)$ : 632 , $1348,1564,1623,2934,3247,3404 ;{ }^{1} \mathrm{H}-\mathrm{NMR}\left(400 \mathrm{MHz}, \mathrm{DMSO}-d_{6}, \delta \mathrm{ppm}\right) 0.99-1.23\left(\mathrm{~m}, 6 \mathrm{H}, \mathrm{CH}_{2}\right)$, 1.50-1.79 (m, 7H, $\left.\mathrm{CH}_{2}\right), 2.30-2.35\left(\mathrm{~m}, 1 \mathrm{H}, \mathrm{CH}_{2}\right), 2.64-2.69\left(\mathrm{~m}, 1 \mathrm{H}, \mathrm{CH}_{2}\right), 3.27-3.33\left(\mathrm{~m}, 1 \mathrm{H}, \mathrm{CH}_{2}\right)$, 3.33-3.39 (m, 1H, CH), 4.95-5.02 (m, 1H, CH), $6.57(\mathrm{~d}, 1 \mathrm{H}, J=8.05 \mathrm{~Hz}, \mathrm{Ar}-\mathrm{H}), 6.61(\mathrm{~s}, 1 \mathrm{H}, \mathrm{Ar}-\mathrm{H}), 6.82$ (d, $1 \mathrm{H}, J=8.13 \mathrm{~Hz}, \mathrm{Ar}-\mathrm{H}), 7.31$ (s, 1H, Ar-H), 7.93 (br s, 1H, NH), 9.83 (br s, 1H, NH), 9.94 (br s, 1H, OH). ${ }^{13} \mathrm{C}-\mathrm{NMR}\left(151 \mathrm{MHz}\right.$, DMSO- $\left.d_{6}, \delta \mathrm{ppm}\right) 23.8,25.3,25.8,33.6,46.8,49.2,55.0,65.5,102.3,110.4$, 114.0, 126.5, 127.1, 128.7, 129.4, 130.6, 133.4, 138.7, 148.3, 154.7, 156.3; Elemental analysis: calc. for $\mathrm{C}_{23} \mathrm{H}_{25} \mathrm{ClN}_{6} \mathrm{O}_{6}$ (516.9): $\mathrm{C} 53.44 ; \mathrm{H} 4.87 ; \mathrm{Cl} 6.86 ; \mathrm{N} 16.26$; found $\mathrm{C} 53.33 ; \mathrm{H} 4.75 ; \mathrm{Cl} 6.79 ; \mathrm{N} 16.21 ; \mathrm{ESI} \mathrm{m} / z$ : $[\mathrm{M}+\mathrm{H}]^{+}$: calc. for $\mathrm{C}_{23} \mathrm{H}_{26} \mathrm{ClN}_{6} \mathrm{O}_{6} 517.9$; found 518 .

6-Chloro-4-((4-(1-((2-(dimethylammonio)ethyl)carbamoyl)pyrrolidin-2-yl)-3-hydroxyphenyl)amino)-5-ni trobenzo[c][1,2,5]oxadiazole 1-oxide 2,2,2-trifluoroacetate (6h). Beige solid, yield 57\%, m.p. $211-212{ }^{\circ} \mathrm{C}$ with decomposition; IR $\left(v, \mathrm{~cm}^{-1}\right)$ : $1349,1566,1623,3175,3275,3400 ;{ }^{1} \mathrm{H}-\mathrm{NMR}\left(400 \mathrm{MHz}\right.$, DMSO- $d_{6}$, $\delta$ ppm) 1.70-1.79 (m, 1H, $\left.\mathrm{CH}_{2}\right), 1.84-1.91\left(\mathrm{~m}, 1 \mathrm{H}, \mathrm{CH}_{2}\right), 2.08-2.19\left(\mathrm{~m}, 1 \mathrm{H}, \mathrm{CH}_{2}\right), 2.79\left(\mathrm{~s}, 6 \mathrm{H}, \mathrm{CH}_{3}\right)$, 3.07-3.13 (m, 2H, $\left.\mathrm{CH}_{2}\right), 3.32-3.38\left(\mathrm{~m}, 3 \mathrm{H}, \mathrm{CH}_{2}\right), 3.51-3.58\left(\mathrm{~m}, 1 \mathrm{H}, \mathrm{CH}_{2}\right), 5.04-5.12(\mathrm{~m}, 1 \mathrm{H}, \mathrm{CH}), 6.54(\mathrm{~d}$, $1 \mathrm{H}, J=7.9 \mathrm{~Hz}, \mathrm{Ar}-\mathrm{H}), 6.58(\mathrm{~s}, 1 \mathrm{H}, \mathrm{Ar}-\mathrm{H}), 6.63(\mathrm{~s}, 1 \mathrm{H}, \mathrm{Ar}-\mathrm{H}), 7.35$ (s, 1H, NH), 9.76 (s, 1H,NH), 9.83 (s, $1 \mathrm{H}, \mathrm{OH}) ;{ }^{13} \mathrm{C}-\mathrm{NMR}\left(151 \mathrm{MHz}, \mathrm{DMSO}-d_{6}, \delta\right.$ ppm) 23.3, 32.9, 36.0, 43.1, 46.7, 55.9, 58.0, 102.3, 110.1, 113.5, 114.3, 114.7, 117.7 (q, $J=300.3 \mathrm{~Hz}), 126.1,127.1,130.5,133.4,138.5,140.3,148.3,154.5,157.1,158.2$, $158.5\left(\mathrm{q}, J=31.0 \mathrm{~Hz}\right.$ ); Elemental analysis: calc. for $\mathrm{C}_{23} \mathrm{H}_{25} \mathrm{ClF}_{3} \mathrm{~N}_{7} \mathrm{O}_{8}$ (619.9): $\mathrm{C}, 44.56 ; \mathrm{H}, 4.06 ; \mathrm{Cl}, 5.72$; $\mathrm{N}, 15.82$; found $\mathrm{C}, 44.79 ; \mathrm{H}, 3.81 ; \mathrm{Cl}, 5.87 ; \mathrm{N}, 15.99 ; \mathrm{ESI} m / z$ : $\left[\mathrm{M}-\mathrm{CF}_{3} \mathrm{CO}_{2}\right]^{+}$: calc. for $\mathrm{C}_{21} \mathrm{H}_{25} \mathrm{ClN}_{7} \mathrm{O}_{6}$ 506.9 ; found 507.

2-(4-Hydroxy-6-methyl-2-oxo-2H-pyran-3-yl)-N-phenylpyrrolidine-1-carboxamide (7b). White solid, yield $69 \%$, m.p. $190-191{ }^{\circ} \mathrm{C}$; IR $\left(v, \mathrm{~cm}^{-1}\right)$ : $1588,1612,1692,2630,2687,2872,2961 ;{ }^{1} \mathrm{H}-\mathrm{NMR}(400 \mathrm{MHz}$, DMSO- $\left.d_{6}, \delta \mathrm{ppm}\right) 1.76-1.87\left(\mathrm{~m}, 1 \mathrm{H}, \mathrm{CH}_{2}\right), 1.95-2.06\left(\mathrm{~m}, 2 \mathrm{H}, \mathrm{CH}_{2}\right), 2.08-2.21\left(\mathrm{~m}, 1 \mathrm{H}, \mathrm{CH}_{2}\right), 2.13(\mathrm{~s}$, $\left.3 \mathrm{H}, \mathrm{CH}_{3}\right), 3.50-3.65\left(\mathrm{~m}, 2 \mathrm{H}, \mathrm{CH}_{2}\right), 4.99-5.09(\mathrm{~m}, 1 \mathrm{H}, \mathrm{CH}), 5.98(\mathrm{~s}, 1 \mathrm{H}, \mathrm{Ar}-\mathrm{H}), 6.88(\mathrm{t}, 1 \mathrm{H}, J=7.3 \mathrm{~Hz}$, $\mathrm{Ar}-\mathrm{H}), 7.18(\mathrm{t}, 2 \mathrm{H}, J=7.8 \mathrm{~Hz}, \mathrm{Ar}-\mathrm{H}), 7.42(\mathrm{~d}, 2 \mathrm{H}, J=8.1 \mathrm{~Hz}, \mathrm{Ar}-\mathrm{H}), 7.81(\mathrm{~s}, 1 \mathrm{H}, \mathrm{NH}), 11.54(\mathrm{~s}, 1 \mathrm{H}, \mathrm{OH})$; ${ }^{13} \mathrm{C}-\mathrm{NMR}\left(151 \mathrm{MHz}, \mathrm{DMSO}-d_{6}, \delta\right.$ ppm) 19.7, 25.4, 30.8, 47.4, 52.1, 100.7, 118.1, 119.4, 121.8, 128.7, 141.1, 153.6, 161.3, 163.8, 166.1; Elemental analysis: calc. for $\mathrm{C}_{17} \mathrm{H}_{18} \mathrm{~N}_{2} \mathrm{O}_{4}$ (314): C, 64.96; H, 5.77; N, 8.91; found $\mathrm{C}, 65.22 ; \mathrm{H}, 5.89 ; \mathrm{N}, 8.80$; $\mathrm{ESI} m / z$ : $[\mathrm{M}+\mathrm{H}]^{+}$: calc. for $\mathrm{C}_{17} \mathrm{H}_{19} \mathrm{~N}_{2} \mathrm{O}_{4} 315$; found 315 .

2-(4-Hydroxy-6-methyl-2-oxo-2H-pyran-3-yl)-N-(4-methoxyphenyl)pyrrolidine-1-carboxamide (7c). White solid, yield $87 \%$, m.p. $168-169^{\circ} \mathrm{C}$; IR $\left(v, \mathrm{~cm}^{-1}\right)$ : $1579,1612,1690,2631,2678,2872,3006 ;{ }^{1} \mathrm{H}-\mathrm{NMR}$ (400 MHz, DMSO- $\left.d_{6}, \delta \mathrm{ppm}\right) 1.75-1.86\left(\mathrm{~m}, 1 \mathrm{H}, \mathrm{CH}_{2}\right), 1.97-2.18\left(\mathrm{~m}, 3 \mathrm{H}, \mathrm{CH}_{2}\right), 2.13\left(\mathrm{~s}, 3 \mathrm{H}, \mathrm{CH}_{3}\right)$, 3.46-3.59 (m, 2H, $\left.\mathrm{CH}_{2}\right), 3.68\left(\mathrm{~s}, 3 \mathrm{H}, \mathrm{CH}_{3}\right), 4.96-5.03(\mathrm{~m}, 1 \mathrm{H}, \mathrm{CH}), 5.97(\mathrm{~s}, 1 \mathrm{H}, \mathrm{Ar}-\mathrm{H}), 6.78(\mathrm{~d}, 2 \mathrm{H}, J=8.8$ $\mathrm{Hz}, \mathrm{Ar}-\mathrm{H}), 7.31$ (d, 2H, J = 9.1 Hz, Ar-H), 7.69 (s, 1H, NH), 11.54 (s, 1H, OH); ${ }^{13} \mathrm{C}-\mathrm{NMR}(151 \mathrm{MHz}$, 
DMSO- $d_{6}, \delta$ ppm) 19.7, 25.4, 30.7, 47.3, 52.1, 55.6, 100.7, 102.9, 114.0, 121.2, 134.1, 154.0, 154.7, 161.3, 163.8, 166.2; Elemental analysis: calc. for $\mathrm{C}_{18} \mathrm{H}_{20} \mathrm{~N}_{2} \mathrm{O}_{5}$ (344): $\mathrm{C}, 62.78 ; \mathrm{H}, 5.85 ; \mathrm{N}, 8.13$; found $\mathrm{C}, 62.89$; $\mathrm{H}, 5.98 ; \mathrm{N}, 8.06$; ESI $m / z$ : $[\mathrm{M}+\mathrm{H}]^{+}$: calc. for $\mathrm{C}_{18} \mathrm{H}_{21} \mathrm{~N}_{2} \mathrm{O}_{5} 345$; found 315 .

$\mathrm{N}$-(4-Bromophenyl)-2-(4-hydroxy-6-methyl-2-oxo-2H-pyran-3-yl)pyrrolidine-1-carboxamide (7d). White solid, yield 75\%, m.p. $194-195^{\circ} \mathrm{C}$; IR $\left(v, \mathrm{~cm}^{-1}\right)$ : 1578, 1612, 1679, 2654, 2815, 2877, 2989; ${ }^{1} \mathrm{H}-\mathrm{NMR}(400$ MHz, DMSO- $d_{6}, \delta$ ppm) 1.76-1.86 (m, $\left.1 \mathrm{H}, \mathrm{CH}_{2}\right), 1.92-2.04\left(\mathrm{~m}, 2 \mathrm{H}, \mathrm{CH}_{2}\right), 2.06-2.17\left(\mathrm{~m}, 1 \mathrm{H}, \mathrm{CH}_{2}\right), 2.12$ (s, 3H, CH $\mathrm{CH}_{3}, 3.50-3.61\left(\mathrm{~m}, 2 \mathrm{H}, \mathrm{CH}_{2}\right), 4.97-5.05(\mathrm{~m}, 1 \mathrm{H}, \mathrm{CH}), 5.96(\mathrm{~s}, 1 \mathrm{H}, \mathrm{Ar}-\mathrm{H}), 7.35(\mathrm{~d}, 2 \mathrm{H}, J=8.7$ $\mathrm{Hz}, \mathrm{Ar}-\mathrm{H}), 7.43$ (d, 2H, J = 8.5 Hz, Ar-H), $8.03(\mathrm{~s}, 1 \mathrm{H}, \mathrm{NH}), 11.42(\mathrm{~s}, 1 \mathrm{H}, \mathrm{OH}) ;{ }^{13} \mathrm{C}-\mathrm{NMR}(151 \mathrm{MHz}$, DMSO- $_{6}, \delta$ ppm) 19.7, 25.4, 30.8, 47.4, 52.3, 100.7, 102.7, 113.1, 121.2, 131.5, 140.6, 153.3, 161.2, 163.7, 166.0; Elemental analysis: calc. for $\mathrm{C}_{17} \mathrm{H}_{17} \mathrm{BrN}_{2} \mathrm{O}_{4}$ (392): $\mathrm{C}, 51.92 ; \mathrm{H}, 4.36 ; \mathrm{Br}, 20.32 ; \mathrm{N}, 7.12$; found $\mathrm{C}$, 52.09; $\mathrm{H}, 4.50 ; \mathrm{Br}, 20.48 ; \mathrm{N}, 7.31$; ESI $m / z:[\mathrm{M}+\mathrm{H}]^{+}$: calc. for $\mathrm{C}_{17} \mathrm{H}_{18} \mathrm{BrN}_{2} \mathrm{O}_{4} 393$; found 393.

$\mathrm{N}$-(4-Fluorophenyl)-2-(4-hydroxy-6-methyl-2-oxo-2H-pyran-3-yl)pyrrolidine-1-carboxamide (7e). White solid, yield 58\%, m.p. $177-178{ }^{\circ} \mathrm{C}$; IR $\left(v, \mathrm{~cm}^{-1}\right)$ : 1602, 1631, 1689, 2631, 2689, 2881, 3066; ${ }^{1} \mathrm{H}-\mathrm{NMR}$ (400 MHz, DMSO- $\left.d_{6}, \delta \mathrm{ppm}\right) 1.74-1.87\left(\mathrm{~m}, 1 \mathrm{H}, \mathrm{CH}_{2}\right), 1.91-2.14\left(\mathrm{~m}, 3 \mathrm{H}, \mathrm{CH}_{2}\right), 2.12\left(\mathrm{~s}, 3 \mathrm{H}, \mathrm{CH}_{3}\right)$, 3.50-3.63 (m, 2H, CH $\mathrm{CH}_{2}, 4.95-5.07(\mathrm{~m}, 1 \mathrm{H}, \mathrm{CH}), 5.97$ (s, 1H, Ar-H), 6.98-7.04 (m, 2H, Ar-H), 7.39-7.49 $(\mathrm{m}, 2 \mathrm{H}, \mathrm{Ar}-\mathrm{H}), 7.92(\mathrm{~s}, 1 \mathrm{H}, \mathrm{NH}), 11.62(\mathrm{~s}, 1 \mathrm{H}, \mathrm{OH}) ;{ }^{13} \mathrm{C}-\mathrm{NMR}\left(151 \mathrm{MHz}, \mathrm{DMSO}-d_{6}, \delta \mathrm{ppm}\right)$ 19.7, 25.4, 30.8, 47.3, 52.2, 100.6, 102.7, $115.1(\mathrm{~d}, J=22.0 \mathrm{~Hz}), 121.0(\mathrm{~d}, J=7.4 \mathrm{~Hz}), 137.4(\mathrm{~d}, J=2.5 \mathrm{~Hz}), 153.6,157.5$ $(\mathrm{d}, J=237.6 \mathrm{~Hz}), 161.2,163.7,166.0$; Elemental analysis: calc. for $\mathrm{C}_{17} \mathrm{H}_{17} \mathrm{FN}_{2} \mathrm{O}_{4}$ (332): C, 61.44; $\mathrm{H}, 5.16$; $\mathrm{N}, 8.43$; found 61.55; $\mathrm{H}, 4.89 ; \mathrm{N}, 8.27$; $\mathrm{ESI} m / z$ : $[\mathrm{M}+\mathrm{H}]^{+}$: calc. for $\mathrm{C}_{17} \mathrm{H}_{18} \mathrm{FN}_{2} \mathrm{O}_{4} 333$; found 333 .

N-Hexyl-2-(4-hydroxy-6-methyl-2-oxo-2H-pyran-3-yl)pyrrolidine-1-carboxamide (7f). White solid, yield 47\%, m.p. 137-138 ${ }^{\circ} \mathrm{C}$; IR $\left(v, \mathrm{~cm}^{-1}\right)$ : 1592, 1690, 2631, 2686, 2935, 3079; ${ }^{1} \mathrm{H}-\mathrm{NMR}\left(400 \mathrm{MHz}\right.$, DMSO- $d_{6}$, $\delta \mathrm{ppm}) 0.84\left(\mathrm{t}, 3 \mathrm{H}, J=7.0 \mathrm{~Hz}, \mathrm{CH}_{3}\right), 1.16-1.27\left(\mathrm{~m}, 6 \mathrm{H}, \mathrm{CH}_{2}\right), 1.30-1.38\left(\mathrm{~m}, 2 \mathrm{H}, \mathrm{CH}_{2}\right), 1.70-1.80(\mathrm{~m}$, $\left.1 \mathrm{H}, \mathrm{CH}_{2}\right), 1.95-2.10\left(\mathrm{~m}, 3 \mathrm{H}, \mathrm{CH}_{2}\right), 2.13\left(\mathrm{~s}, 3 \mathrm{H}, \mathrm{CH}_{3}\right), 2.87-2.96\left(\mathrm{~m}, 1 \mathrm{H}, \mathrm{CH}_{2}\right), 2.98-3.07\left(\mathrm{~m}, 1 \mathrm{H}, \mathrm{CH}_{2}\right)$, 3.31-3.35 (m, 1H, $\left.\mathrm{CH}_{2}\right), 3.36-3.41\left(\mathrm{~m}, 1 \mathrm{H}, \mathrm{CH}_{2}\right), 4.80-4.88(\mathrm{~m}, 1 \mathrm{H}, \mathrm{CH}), 5.70(\mathrm{~s}, 1 \mathrm{H}, \mathrm{NH}), 5.96(\mathrm{~s}, 1 \mathrm{H}$, Ar-H); ${ }^{13}$ C-NMR (151 MHz, DMSO- $d_{6}, \delta$ ppm) $14.4,19.7,22.5,25.3,26.5,30.3,30.4,31.5,35.6,47.0,51.9$, 101.0, 103.0, 156.9, 161.4, 163.5, 167.0; Elemental analysis: calc. for $\mathrm{C}_{17} \mathrm{H}_{26} \mathrm{~N}_{2} \mathrm{O}_{4}$ (322): C, 63.33; $\mathrm{H}, 8.13$; $\mathrm{N}$, 8.69; found $\mathrm{C}, 63.50 ; \mathrm{H}, 8.31 ; \mathrm{N}, 8.87$; $\mathrm{ESI} m / z:[\mathrm{M}+\mathrm{H}]^{+}$: calc. for $\mathrm{C}_{17} \mathrm{H}_{27} \mathrm{~N}_{2} \mathrm{O}_{4} 323$; found 323 .

$\mathrm{N}$-Cyclohexyl-2-(4-hydroxy-6-methyl-2-oxo-2H-pyran-3-yl)pyrrolidine-1-carboxamide (7g). Beige solid, yield $60 \%$, m.p. $184-185^{\circ} \mathrm{C}$; IR $\left(v, \mathrm{~cm}^{-1}\right)$ : $1593,1692,2631,2686,2934,3079 ;{ }^{1} \mathrm{H}-\mathrm{NMR}(400 \mathrm{MHz}$, DMSO- $d_{6}, \delta$ ppm) 0.99-1.14 (m, 3H, $\left.\mathrm{CH}_{2}\right), 1.16-1.28\left(\mathrm{~m}, 2 \mathrm{H}, \mathrm{CH}_{2}\right), 1.48-1.54\left(\mathrm{~m}, 1 \mathrm{H}, \mathrm{CH}_{2}\right), 1.55-1.66$ $\left(\mathrm{m}, 3 \mathrm{H}, \mathrm{CH}_{2}\right), 1.69-1.77\left(\mathrm{~m}, 2 \mathrm{H}, \mathrm{CH}_{2}\right), 1.99-2.09\left(\mathrm{~m}, 3 \mathrm{H}, \mathrm{CH}_{2}\right), 2.12-2.22\left(\mathrm{~m}, 1 \mathrm{H}, \mathrm{CH}_{2}\right), 2.14(\mathrm{~s}, 3 \mathrm{H}$, $\left.\mathrm{CH}_{3}\right), 3.36-3.39\left(\mathrm{~m}, 1 \mathrm{H}, \mathrm{CH}_{2}\right), 3.40-3.47\left(\mathrm{~m}, 1 \mathrm{H}, \mathrm{CH}_{2}\right), 4.78-4.87(\mathrm{~m}, 1 \mathrm{H}, \mathrm{CH}), 5.36(\mathrm{~s}, 1 \mathrm{H}, \mathrm{NH}), 5.97(\mathrm{~s}$, 1H, Ar-H), 12.01 (s, 1H, OH); ${ }^{13} \mathrm{C}-\mathrm{NMR}\left(151 \mathrm{MHz}, \mathrm{DMSO}-d_{6}, \delta\right.$ ppm) 19.7, 25.1, 25.3, 25.8, 30.3, 33.6, 47.1, 49.1, 51.6, 100.8, 102.8, 156.1, 161.7, 163.6, 167.0; Elemental analysis: calc. for $\mathrm{C}_{17} \mathrm{H}_{24} \mathrm{~N}_{2} \mathrm{O}_{4}$ (320): C, 63.73; $\mathrm{H}, 7.55 ; \mathrm{N}, 8.74$; found C, 63.87; $\mathrm{H}, 7.76 ; \mathrm{N}, 8.59$; ESI m/z: $[\mathrm{M}+\mathrm{H}]^{+}$: calc. for $\mathrm{C}_{17} \mathrm{H}_{25} \mathrm{~N}_{2} \mathrm{O}_{4} 321$; found 321.

2-(2-(4-Hydroxy-6-methyl-2-oxo-2H-pyran-3-yl)pyrrolidine-1-carboxamido)- $\mathrm{N}, \mathrm{N}$-dimethylethan-1-aminium 2,2,2-trifluoroacetate (7h). White solid, yield $77 \%$, m.p. $146-147^{\circ} \mathrm{C} ; \operatorname{IR~}\left(\mathrm{v}, \mathrm{cm}^{-1}\right): 1592,1692,2683,2985$, $3064 ;{ }^{1} \mathrm{H}-\mathrm{NMR}\left(400 \mathrm{MHz}, \mathrm{DMSO}-d_{6}, \delta \mathrm{ppm}\right) 1.73-1.81\left(\mathrm{~m}, 1 \mathrm{H}, \mathrm{CH}_{2}\right), 1.83-1.90\left(\mathrm{~m}, 1 \mathrm{H}, \mathrm{CH}_{2}\right), 1.96-2.08$ $\left(\mathrm{m}, 2 \mathrm{H}, \mathrm{CH}_{2}\right), 2.12\left(\mathrm{~s}, 3 \mathrm{H}, \mathrm{CH}_{3}\right), 2.78\left(\mathrm{~s}, 6 \mathrm{H}, \mathrm{CH}_{3}\right), 3.05-3.12\left(\mathrm{~m}, 2 \mathrm{H}, \mathrm{CH}_{2}\right), 3.21-3.30\left(\mathrm{~m}, 1 \mathrm{H}, \mathrm{CH}_{2}\right)$, 3.32-3.39 (m, 3H, CH $), 4.85-4.94(\mathrm{~m}, 1 \mathrm{H}, \mathrm{CH}), 5.98(\mathrm{~s}, 1 \mathrm{H}, \mathrm{Ar}-\mathrm{H}), 6.22(\mathrm{~s}, 1 \mathrm{H}, \mathrm{NH}), 9.56(\mathrm{~s}, 1 \mathrm{H}, \mathrm{OH})$, $11.70\left(\mathrm{~s}, 1 \mathrm{H}, \mathrm{NH}^{+}\right) ;{ }^{13} \mathrm{C}-\mathrm{NMR}\left(151 \mathrm{MHz}, \mathrm{DMSO}-d_{6}, \delta \mathrm{ppm}\right) 19.7,25.2,31.3,36.0,43.1,46.9,52.4,58.1$, 100.8, 102.9, $177.7(\mathrm{q}, J=31.2 \mathrm{~Hz}), 156.8,158.6(\mathrm{q}, J=299.4 \mathrm{~Hz}), 160.9,163.6,166.2$; Elemental analysis: calc. for $\mathrm{C}_{17} \mathrm{H}_{24} \mathrm{~F}_{3} \mathrm{~N}_{3} \mathrm{O}_{6}$ (423): $\mathrm{C}, 48.23 ; \mathrm{H}, 5.71 ; \mathrm{N}, 9.92$; found $\mathrm{C}, 48.30 ; \mathrm{H}, 5.85 ; \mathrm{N}, 10.14 ; \mathrm{ESI} \mathrm{m} / \mathrm{z}$ : $\mathrm{M}-$ $\left.\mathrm{CF}_{3} \mathrm{CO}_{2}\right]^{+}$: calc. for $\mathrm{C}_{15} \mathrm{H}_{24} \mathrm{~N}_{3} \mathrm{O}_{4} 310$; found 310 .

2-(4-Hydroxy-2-oxo-2H-chromen-3-yl)-N-phenylpyrrolidine-1-carboxamide (8b). Beige solid, yield 51\%, m.p. $179-180{ }^{\circ} \mathrm{C}$; IR $\left(v, \mathrm{~cm}^{-1}\right)$ : $1595,1616,1695,2853,2930,3075 .{ }^{1} \mathrm{H}-\mathrm{NMR}\left(400 \mathrm{MHz}, \mathrm{DMSO}-d_{6}, \delta\right.$ ppm) 1.87-1.98 (m, 1H, CH $)$, 2.13-2.27 (m, 3H, $\left.\mathrm{CH}_{2}\right), 3.64-3.71\left(\mathrm{~m}, 2 \mathrm{H}, \mathrm{CH}_{2}\right), 5.23-5.29(\mathrm{~m}, 1 \mathrm{H}, \mathrm{CH})$, $6.91(\mathrm{t}, 1 \mathrm{H}, J=7.4 \mathrm{~Hz}, \mathrm{Ar}-\mathrm{H}), 7.19(\mathrm{t}, 3 \mathrm{H}, J=7.9 \mathrm{~Hz}, \mathrm{Ar}-\mathrm{H}), 7.32-7.37(\mathrm{~m}, 2 \mathrm{H}, \mathrm{Ar}-\mathrm{H}, \mathrm{NH}), 7.42(\mathrm{~d}, 2 \mathrm{H}, J$ $=8.0 \mathrm{~Hz}, \mathrm{Ar}-\mathrm{H}), 7.59(\mathrm{t}, 1 \mathrm{H}, J=8.0 \mathrm{~Hz}, \mathrm{Ar}-\mathrm{H}), 7.94(\mathrm{~d}, 1 \mathrm{H}, J=7.9 \mathrm{~Hz}, \mathrm{Ar}-\mathrm{H}), 8.26(\mathrm{~s}, 1 \mathrm{H}, \mathrm{OH}) ;{ }^{13} \mathrm{C}-\mathrm{NMR}$ 
(151 MHz, DMSO- $d_{6}, \delta$ ppm) 25.8, 29.6, 47.8, 53.2, 106.5, 116.5, 118.1, 120.2, 122.3, 124.0, 124.3, 128.7, 132.5, 140.6, 152.7, 154.9, 161.3, 162.2; Elemental analysis: calc. for $\mathrm{C}_{20} \mathrm{H}_{18} \mathrm{~N}_{2} \mathrm{O}_{4}$ (350): C, 68.56; $\mathrm{H}, 5.18$; $\mathrm{N}$, 8.00; found C, 68.70; $\mathrm{H}, 5.40 ; \mathrm{N}, 7.89 ; \mathrm{ESI} \mathrm{m} / \mathrm{z}:[\mathrm{M}+\mathrm{H}]^{+}$: calc. for $\mathrm{C}_{20} \mathrm{H}_{19} \mathrm{~N}_{2} \mathrm{O}_{4} 351$; found 351 .

2-(4-Hydroxy-2-oxo-2H-chromen-3-yl)-N-(4-methoxyphenyl)pyrrolidine-1-carboxamide (8c). Beige solid, yield $45 \%$, m.p. $152-153{ }^{\circ} \mathrm{C}$; IR $\left(v, \mathrm{~cm}^{-1}\right)$ : $1596,1617,1697,2847,2984,3036 ;{ }^{1} \mathrm{H}-\mathrm{NMR}(400 \mathrm{MHz}$, DMSO- $\left.d_{6}, \delta \mathrm{ppm}\right) 1.81-2.00\left(\mathrm{~m}, 1 \mathrm{H}, \mathrm{CH}_{2}\right), 2.12-2.23\left(\mathrm{~m}, 2 \mathrm{H}, \mathrm{CH}_{2}\right), 2.24-2.34\left(\mathrm{~m}, 1 \mathrm{H}, \mathrm{CH}_{2}\right), 3.59-3.66$ $\left(\mathrm{m}, 2 \mathrm{H}, \mathrm{CH}_{2}\right), 3.69\left(\mathrm{~s}, 3 \mathrm{H}, \mathrm{CH}_{3}\right), 5.18-5.28(\mathrm{~m}, 1 \mathrm{H}, \mathrm{CH}), 6.79(\mathrm{~d}, 2 \mathrm{H}, J=9.1 \mathrm{~Hz}, \mathrm{Ar}-\mathrm{H}), 7.25-7.38(\mathrm{~m}, 5 \mathrm{H}$, Ar-H, NH), 7.59 (t, 1H, J = 7.0 Hz, Ar-H), $7.92(\mathrm{~d}, 1 \mathrm{H}, J=6.7 \mathrm{~Hz}, \mathrm{Ar}-\mathrm{H}), 8.19$ (s, 1H, OH); ${ }^{13} \mathrm{C}-\mathrm{NMR}(151$ MHz, DMSO- $d_{6}, \delta$ ppm) $25.8,29.4,47.7,53.2,55.6,106.5,114.0,116.4,120.4,122.3,123.9,124.3,132.5$, 133.3, 152.7, 155.1, 155.5, 161.2, 162.6; Elemental analysis: calc. for $\mathrm{C}_{21} \mathrm{H}_{20} \mathrm{~N}_{2} \mathrm{O}_{5}$ (380): C, 66.53; $\mathrm{H}, 5.50$; $\mathrm{N}$, 7.49; found $\mathrm{C}$, 66.53; $\mathrm{H}, 5.50 ; \mathrm{N}, 7.49$; $\mathrm{ESI} m / z:[\mathrm{M}+\mathrm{H}]^{+}$: calc. for $\mathrm{C}_{21} \mathrm{H}_{21} \mathrm{~N}_{2} \mathrm{O}_{5} 381$; found 381 .

$\mathrm{N}$-(4-Bromophenyl)-2-(4-hydroxy-2-oxo-2H-chromen-3-yl)pyrrolidine-1-carboxamide (8d). Beige solid, yield $68 \%$, m.p. $179{ }^{\circ} \mathrm{C}$; IR $\left(v, \mathrm{~cm}^{-1}\right)$ : $1595,1617,2797,2837,2987,3078 ;{ }^{1} \mathrm{H}-\mathrm{NMR}\left(400 \mathrm{MHz}\right.$, DMSO- $d_{6}$, $\delta$ ppm) 1.83-1.86 (m, 1H, CH $), 2.08-2.27\left(\mathrm{~m}, 3 \mathrm{H}, \mathrm{CH}_{2}\right), 3.62-3.72\left(\mathrm{~m}, 2 \mathrm{H}, \mathrm{CH}_{2}\right), 5.23-5.31(\mathrm{~m}, 1 \mathrm{H}, \mathrm{CH})$, 7.30-7.40 (m, 5H, Ar-H, NH), $7.43(\mathrm{~d}, 2 \mathrm{H}, J=8.90 \mathrm{~Hz}), 7.59(\mathrm{t}, 1 \mathrm{H}, J=8.2 \mathrm{~Hz}, \mathrm{Ar}-\mathrm{H}), 7.94(\mathrm{~d}, 1 \mathrm{H}, J=8.3$ $\mathrm{Hz}, \mathrm{Ar}-\mathrm{H}), 8.39$ (s, $1 \mathrm{H}, \mathrm{OH}) ;{ }^{13} \mathrm{C}-\mathrm{NMR}\left(151 \mathrm{MHz}, \mathrm{DMSO}-d_{6}, \delta \mathrm{ppm}\right) 25.8,29.8,47.8,53.2,106.5,113.6$, 116.5, 120.0, 121.8, 123.9, 124.3, 131.5, 132.4, 140.2, 152.7, 154.3, 161.3, 161.9; Elemental analysis: calc. for $\mathrm{C}_{20} \mathrm{H}_{17} \mathrm{BrN}_{2} \mathrm{O}_{4}$ (429): $\mathrm{C}, 55.96 ; \mathrm{H}, 3.99 ; \mathrm{Br}, 18.61 ; \mathrm{N}, 6.53$; found $\mathrm{C}, 56.14 ; \mathrm{H}, 4.18 ; \mathrm{Br}, 18.73 ; \mathrm{N}, 6.70$; $\mathrm{ESI}$ $m / z:[\mathrm{M}+\mathrm{H}]^{+}$: calc. for $\mathrm{C}_{20} \mathrm{H}_{18} \mathrm{BrN}_{2} \mathrm{O}_{4} 430$; found 430 .

N-(4-Fluorophenyl)-2-(4-hydroxy-2-oxo-2H-chromen-3-yl)pyrrolidine-1-carboxamide (8e). Beige solid, yield 51\%, m.p. $165^{\circ} \mathrm{C}$; IR $\left(v, \mathrm{~cm}^{-1}\right)$ : 1589, 1624, 1697, 2847, 2983, 3036, 3106; ${ }^{1} \mathrm{H}-\mathrm{NMR}(400 \mathrm{MHz}$, DMSO- $d_{6}, \delta$ ppm) 1.92-1.98 (m, $\left.1 \mathrm{H}, \mathrm{CH}_{2}\right), 1.12-1.28\left(\mathrm{~m}, 3 \mathrm{H}, \mathrm{CH}_{2}\right), 3.61-3.70\left(\mathrm{~m}, 2 \mathrm{H}, \mathrm{CH}_{2}\right), 5.22-5.29$ (m, 1H, CH), 6.99-7.05 (m, 2H, Ar-H), 7.29-7.40 (m, 3H, Ar-H, NH), 7.40-7.47 (m, 2H, Ar-H), 7.59 (t, $1 \mathrm{H}, J=8.5 \mathrm{~Hz}, \mathrm{Ar}-\mathrm{H}), 7.93(\mathrm{~d}, 1 \mathrm{H}, J=6.9 \mathrm{~Hz}, \mathrm{Ar}-\mathrm{H}), 8.33(\mathrm{~s}, 1 \mathrm{H}, \mathrm{OH}) ;{ }^{13} \mathrm{C}-\mathrm{NMR}\left(151 \mathrm{MHz}, \mathrm{DMSO}-d_{6}, \delta\right.$ ppm) 25.7, 29.6, 47.7, 53.1, 106.5, $115.2(\mathrm{~d}, J=22.0 \mathrm{~Hz}), 116.4,117.0,121.9(\mathrm{~d}, J=7.7 \mathrm{~Hz}), 123.9,124.2$, 132.4, $136.9(\mathrm{~d}, J=2.5 \mathrm{~Hz}), 152.7,154.8,157.8(\mathrm{~d}, J=138.1 \mathrm{~Hz}), 161.3,162.2$; Elemental analysis: calc. for $\mathrm{C}_{20} \mathrm{H}_{17} \mathrm{FN}_{2} \mathrm{O}_{4}$ (368): C, 65.21; H, 4.65; N, 7.60; found C, 65.42; H, 4.78; N, 7.83; ESI m/z: $[\mathrm{M}+\mathrm{H}]^{+}$: calc. for $\mathrm{C}_{20} \mathrm{H}_{18} \mathrm{FN}_{2} \mathrm{O}_{4} 369$; found 369 .

$\mathrm{N}$-Hexyl-2-(4-hydroxy-2-oxo-2H-chromen-3-yl)pyrrolidine-1-carboxamide (8f). Beige solid, yield 34\%, m.p. $124-125^{\circ} \mathrm{C}$; IR $\left(v, \mathrm{~cm}^{-1}\right)$ : 1554, 1614, 1687, 2858, 2929, 2953, 3075, 3374; ${ }^{1} \mathrm{H}-\mathrm{NMR}(400 \mathrm{MHz}$, DMSO- $\left.d_{6}, \delta \mathrm{ppm}\right) 0.79\left(\mathrm{t}, 3 \mathrm{H}, J=6.8 \mathrm{~Hz}, \mathrm{CH}_{3}\right), 1.19-1.22\left(\mathrm{~m}, 5 \mathrm{H}, \mathrm{CH}_{2}\right), 1.30-1.35\left(\mathrm{~m}, 1 \mathrm{H}, \mathrm{CH}_{2}\right), 1.37-1.41$ $\left(\mathrm{m}, 1 \mathrm{H}, \mathrm{CH}_{2}\right), 1.85-1.95\left(\mathrm{~m}, 1 \mathrm{H}, \mathrm{CH}_{2}\right), 1.07-1.16\left(\mathrm{~m}, 1 \mathrm{H}, \mathrm{CH}_{2}\right), 2.20-2.17\left(\mathrm{~m}, 1 \mathrm{H}, \mathrm{CH}_{2}\right), 2.40-2.48(\mathrm{~m}$, $\left.1 \mathrm{H}, \mathrm{CH}_{2}\right), 2.94-3.02\left(\mathrm{~m}, 2 \mathrm{H}, \mathrm{CH}_{2}\right), 3.03-3.10\left(\mathrm{~m}, 1 \mathrm{H}, \mathrm{CH}_{2}\right), 3.31-3.39\left(\mathrm{~m}, 1 \mathrm{H}, \mathrm{CH}_{2}\right), 3.41-3.49(\mathrm{~m}, 1 \mathrm{H}$, $\left.\mathrm{CH}_{2}\right), 5.06-5.14(\mathrm{~m}, 1 \mathrm{H}, \mathrm{CH}), 6.57(\mathrm{~s}, 1 \mathrm{H}, \mathrm{NH}) ; 7.29-7.33(\mathrm{~m}, 2 \mathrm{H}, \mathrm{Ar}-\mathrm{H}), 7.55-7.60(\mathrm{~m}, 1 \mathrm{H}, \mathrm{Ar}-\mathrm{H}), 7.87$ $(\mathrm{d}, 1 \mathrm{H}, J=7.8 \mathrm{~Hz}, \mathrm{Ar}-\mathrm{H}) ;{ }^{13} \mathrm{C}-\mathrm{NMR}\left(151 \mathrm{MHz}, \mathrm{DMSO}-d_{6}, \delta \mathrm{ppm}\right) 14.3,22.5,25.7,26.5,28.8,30.1,31.5$, 40.7, 47.4, 53.4, 106.3, 116.3, 117.2, 124.1, 124.2, 132.6, 153.0, 158.8, 161.1, 164.5; Elemental analysis: calc. for $\mathrm{C}_{20} \mathrm{H}_{26} \mathrm{~N}_{2} \mathrm{O}_{4}$ (358): C, 67.02; H, 7.31; N, 7.82; found $\mathrm{C}, 67.29 ; \mathrm{H}, 7.55 ; \mathrm{N}, 7.99$; ESI $m / z$ : $[\mathrm{M}+\mathrm{H}]^{+}$: calc. for $\mathrm{C}_{20} \mathrm{H}_{27} \mathrm{~N}_{2} \mathrm{O}_{4} 359$; found 359 .

Crystal data: $\mathrm{C}_{20} \mathrm{H}_{26} \mathrm{~N}_{2} \mathrm{O}_{4}, \mathrm{M}=358.43$, colorless crystal $0.12 \times 0.15 \times 0.15 \mathrm{~mm}^{3}$, triclinic, space group P-1, $Z=6, a=12.9336(12), b=14.2803(13), c=16.0108(14) \AA, \alpha=70.825(2), \beta=84.411(2), \gamma=$ $87.932(2)^{\circ}, V=2779.8(4) \AA^{3}, \rho_{\text {calc }}=1.285 \mathrm{~g} / \mathrm{cm}^{3}, \mu=0.9 \mathrm{~mm}^{-1}, 33,903$ reflections collected $( \pm \mathrm{h}, \pm \mathrm{k}, \pm \mathrm{l})$, 14,791 independent (Rint 0.0849 ) and 7433 observed reflections $[I \geq 2 \sigma(I)], 703$ refined parameters, $R=$ $0.0673, w R_{2}=0.1723$, max. residual electron density was $0.667(-0.528) \mathrm{e}^{-3}$.

$\mathrm{N}$-Cyclohexyl-2-(4-hydroxy-2-oxo-2H-chromen-3-yl)pyrrolidine-1-carboxamide (8g). Beige solid, yield 68\%, m.p. $166-167^{\circ} \mathrm{C}$; IR $\left(v, \mathrm{~cm}^{-1}\right)$ : 1549, 1616, 1694, 2475, 2853, 2935, 3075, 3373; ${ }^{1} \mathrm{H}-\mathrm{NMR}(400 \mathrm{MHz}$, DMSO- $\left.d_{6}, \delta \mathrm{ppm}\right) 1.01-1.10\left(\mathrm{~m}, 1 \mathrm{H}, \mathrm{CH}_{2}\right), 1.14-1.26\left(\mathrm{~m}, 4 \mathrm{H}, \mathrm{CH}_{2}\right), 1.48-1.56\left(\mathrm{~m}, 1 \mathrm{H}, \mathrm{CH}_{2}\right), 1.59-1.67$ $\left(\mathrm{m}, 2 \mathrm{H}, \mathrm{CH}_{2}\right), 1.68-1.78\left(\mathrm{~m}, 2 \mathrm{H}, \mathrm{CH}_{2}\right), 1.84-1.94\left(\mathrm{~m}, 1 \mathrm{H}, \mathrm{CH}_{2}\right), 2.06-2.16\left(\mathrm{~m}, 1 \mathrm{H}, \mathrm{CH}_{2}\right), 2.20-2.29(\mathrm{~m}$, $\left.1 \mathrm{H}, \mathrm{CH}_{2}\right), 2.39-2.48\left(\mathrm{~m}, 1 \mathrm{H}, \mathrm{CH}_{2}\right), 3.35-3.50\left(\mathrm{~m}, 3 \mathrm{H}, \mathrm{CH}_{2}, \mathrm{CH}\right), 5.04-5.13(\mathrm{~m}, 1 \mathrm{H}, \mathrm{CH}), 6.26(\mathrm{~s}, 1 \mathrm{H}, \mathrm{OH})$, $7.30-7.35(\mathrm{~m}, 2 \mathrm{H}, \mathrm{Ar}-\mathrm{H}), 7.59(\mathrm{td}, 1 \mathrm{H}, J=7.8 \mathrm{~Hz}, J=1.6 \mathrm{~Hz}, \mathrm{Ar}-\mathrm{H}) 7.88(\mathrm{dd}, 1 \mathrm{H}, J=8.3 \mathrm{~Hz}, J=1.6 \mathrm{~Hz}$, Ar-H); ${ }^{13}$ C-NMR (151 MHz, DMSO- $d_{6}, \delta$ ppm) $25.4,25.7,28.7,33.3,33.5,47.5,49.9,53.4,106.2,116.3$, 
117.2, 124.1, 124.2, 132.6, 153.0, 158.1, 161.1, 164.5; Elemental analysis: calc. for $\mathrm{C}_{20} \mathrm{H}_{24} \mathrm{~N}_{2} \mathrm{O}_{4}$ (356): $\mathrm{C}$, 67.40; $\mathrm{H}, 6.79 ; \mathrm{N}, 7.86$; found $\mathrm{C}, 67.54 ; \mathrm{H}, 6.89 ; \mathrm{N}, 7.73$; ESI m/z: $[\mathrm{M}+\mathrm{H}]^{+}$: calc. for $\mathrm{C}_{20} \mathrm{H}_{25} \mathrm{~N}_{2} \mathrm{O}_{4}$ 357; found 357.

2-(2-(4-Hydroxy-2-oxo-2H-chromen-3-yl)pyrrolidine-1-carboxamido)-N,N-dimethylethan-1-aminium 2,2,2-trifluoroacetate (8h). Beige solid, yield 73\%, m.p. 147-148 ${ }^{\circ} \mathrm{C}$; IR $\left(v, \mathrm{~cm}^{-1}\right): 1544,1615,1684,2718$, $2876,2957,3038,3368 ;{ }^{1} \mathrm{H}-\mathrm{NMR}\left(400 \mathrm{MHz}, \mathrm{DMSO}-d_{6}, \delta \mathrm{ppm}\right) 1.83-1.94\left(\mathrm{~m}, 1 \mathrm{H}, \mathrm{CH}_{2}\right), 2.08-2.22(\mathrm{~m}$, $\left.2 \mathrm{H}, \mathrm{CH}_{2}\right), 2.24-2.35\left(\mathrm{~m}, 1 \mathrm{H}, \mathrm{CH}_{2}\right), 2.78\left(\mathrm{~s}, 6 \mathrm{H}, \mathrm{CH}_{3}\right), 3.06-3.15\left(\mathrm{~m}, 2 \mathrm{H}, \mathrm{CH}_{2}\right), 3.26-3.33\left(\mathrm{~m}, 1 \mathrm{H}, \mathrm{CH}_{2}\right)$, 3.35-3.41 (m, 1H, $\left.\mathrm{CH}_{2}\right), 3.43-3.49\left(\mathrm{~m}, 2 \mathrm{H}, \mathrm{CH}_{2}\right), 5.08-5.16(\mathrm{~m}, 1 \mathrm{H}, \mathrm{CH}), 6.75(\mathrm{~s}, 1 \mathrm{H}, \mathrm{NH}), 7.29-7.38(\mathrm{~m}$, 2H, Ar-H), 7.59 (t, 1H, J = 8.2 Hz, Ar-H) $7.92(\mathrm{~d}, 1 \mathrm{H}, J=7.9 \mathrm{~Hz}, \mathrm{Ar}-\mathrm{H}) 9.62(\mathrm{~s}, 1 \mathrm{H}, \mathrm{OH}) ;{ }^{13} \mathrm{C}-\mathrm{NMR}$ (151 MHz, DMSO- $d_{6}, \delta$ ppm) 25.6, 29.7, 36.0, 43.0, 47.2, 53.3, 57.4, 106.2, 116.2, 116.9 (q, $\left.J=199.9 \mathrm{~Hz}\right)$, $117.4,124.1,124.2,132.4,152.9,157.9,158.7(\mathrm{q}, J=32.1 \mathrm{~Hz}), 161.3,163.5$; Elemental analysis: calc. for $\mathrm{C}_{20} \mathrm{H}_{24} \mathrm{~F}_{3} \mathrm{~N}_{3} \mathrm{O}_{6}$ (458): C, 52.29; $\mathrm{H}, 5.27 ; \mathrm{N}, 9.15$; found $\mathrm{C}, 52.48 ; \mathrm{H}, 5.16 ; \mathrm{N}, 8.97 ; \mathrm{ESI} m / z:[\mathrm{M}+\mathrm{H}]^{+}$: calc. for $\mathrm{C}_{18} \mathrm{H}_{24} \mathrm{~N}_{3} \mathrm{O}_{4} 346$; found 346 .

\subsection{Biological Studies}

\subsubsection{In Vitro Studies of Anti-Cancer Activity}

Cytotoxicity assay. Cytotoxic effects of the test compounds on human cancer and normal cells were estimated by means of the multifunctional Cytell Cell Imaging system (GE Health Care Life Science, Sweden) using the Cell Viability Bio App which precisely counts the number of cells and evaluates their viability from fluorescence intensity data. Two fluorescent dyes that selectively penetrate the cell membranes and fluoresce at different wavelengths were used in the experiments. A low-molecular-weight $4^{\prime}, 6$-diamidin-2-phenylindol dye (DAPI) is able to penetrate the intact membranes of living cells and color nuclei in blue. The high-molecular-weight propidium iodide dye penetrates only dead cells with damaged membranes, staining them in yellow. As a result, living cells are painted in blue and dead cells are painted in yellow. DAPI and propidium iodide were purchased from Sigma. The M-Hela clone 11 human, epithelioid cervical carcinoma, strain of Hela, clone of M-Hela from the Type Culture Collection of the Institute of Cytology (Russian Academy of Sciences) and Chang liver cell line (Human liver cells) from N. F. Gamaleya Research Center of Epidemiology and Microbiology were used in the experiments. The cells were cultured in a standard Eagle's nutrient medium manufactured at the Chumakov Institute of Poliomyelitis and Virus Encephalitis (PanEco company) and supplemented with $10 \%$ fetal calf serum and $1 \%$ nonessential amino acids. The cells were plated into a 96-well plate (Eppendorf) at a concentration of 100,000 cells $/ \mathrm{mL}, 150 \mu \mathrm{L}$ of medium per well, and cultured in a $\mathrm{CO}_{2}$ incubator at $37^{\circ} \mathrm{C}$. Twenty-four hours after seeding the cells into wells, the compound under study was added at a preset dilution, $150 \mu \mathrm{L}$ to each well. The dilutions of the compounds at concentrations of 1-100 $\mu \mathrm{M}$ were prepared immediately in nutrient media; $5 \%$ DMSO (which does not induce the inhibition of cells at this concentration) was added for better solubility. The experiments were repeated three times. Intact cells cultured in parallel with experimental cells were used as a control.

Induction of Apoptotic Effects by test compounds. Cell Culture. M-Hela cells at $1 \times 10^{6}$ cells/well in a final volume of $2 \mathrm{~mL}$ were seeded into six-well plates. After 24 hours of incubation, a solution of the test compound $6 \mathrm{~g}$ was added to the wells at the concentration studied.

Cytell Cell Imaging System Assay. M-Hela cells were plated into a 24-well plate (Eppendorf) at a concentration of $1 \times 10^{6}$ cells $/ \mathrm{mL}, 500 \mu \mathrm{L}$ of medium per well, and cultured in a $\mathrm{CO}_{2}$ incubator at $37{ }^{\circ} \mathrm{C}$. Twenty-four hours after seeding the cells into wells the compound was added at a preset dilution, $500 \mu \mathrm{L}$ to each well. The dilutions of compound $6 \mathrm{~g}$ were prepared immediately in nutrient media; $5 \%$ DMSO (which did not induce the inhibition of cells at this concentration) was added for better solubility. Evaluation of apoptotic effects was performed with the help of multifunctional system Cytell Cell Imaging, using Cell Viability BioApp and Automated Imaging BioApp applications. The annexin V-Alexa Fluor 647 apoptosis detection kit, DAPI and propidium iodide purchased from Sigma. 
Multiplex analysis of early apoptosis markers. M-Hela cells were incubated for 24 hours with the test substance. Cells were lysed in MILLIPLEX ${ }^{\circledR}$ MAP Lysis buffer containing protease inhibitors. Twenty micrograms of total protein of each lysate diluted in MILLIPLEX ${ }^{\circledR}$ MAP Assay Buffer 2 was analyzed according to the analysis protocol (the lysate was incubated at $4{ }^{\circ} \mathrm{C}$ overnight). The mean fluorescence intensity (MFI) was detected using the Luminex ${ }^{\circledR}$ system, MERCK, USA.

Flow Cytometry Assay. Mitochondrial membrane potential. Cells were harvested at $2000 \mathrm{rpm}$ for $5 \mathrm{~min}$ and then washed twice with ice-cold PBS, followed by resuspension in JC-10 $(10 \mu \mathrm{g} / \mathrm{mL})$ and incubation at $37^{\circ} \mathrm{C}$ for $10 \mathrm{~min}$. After the cells were rinsed three times and suspended in PBS, the JC-10 fluorescence was observed by flow cytometry (Guava easy Cyte 8HT, Guava Technologies Inc., Hayward, CA, USA).

Statistical analysis. The experiments were repeated three times. The cytometric results were analyzed by the Cytell Cell Imaging multifunctional system using the Cell Viability BioApp and Apoptosis BioApp application. The data in the tables and graphs are given as the mean \pm standard error.

\subsubsection{In Vivo Studies of Anti-Cancer Activity}

Animals. In vivo experiments were performed using the $\mathrm{BDF}_{1}$ hybrid male mice of $22-24 \mathrm{~g}$ weight. The experimental animals were caged in a standard vivarium in $12 \mathrm{~h}$ light conditions with free access to food and water. All manipulations with the animals were performed in accordance with the solutions of the Commission on Bioethics of the Institute of Problems of Chemical Physics, Russian Academy of Sciences (IPCP RAS).

Anti-tumor activity. The tumors were transplanted intraperitoneally (i.p.) in accordance with a standard procedure inoculum: $10^{6}$ tumor cells in isotonic solution of $\mathrm{NaCl}, \mathrm{V}=0.2 \mathrm{~cm}^{3}$ (leukemia P388) [60]. Original compounds were injected intraperitoneally as aqueous solution. Doses from 18 to $83 \mathrm{mg} / \mathrm{kg} /$ day and the mode of administration on days 1, 5, and 9 after transplantation were used. In each experiment, a single group of tumor-bearing animals not injected with the compounds served as the control group. Each group consisted of six mice. The animals were observed daily for survival for a minimum of 60 days. The efficacy of the therapy against leukemia (defined as increase in lifespan-ILS) was assessed as the percentage of the median survival time (MST) of the treated group (t) to that of the control group (c): ILS $(\%)=($ MSTt $/ M S T c) \times 100$.

Statistics. The experiments were carried out in triplicate. The data are presented in the form $X \pm S D$ (mean \pm standard deviation). The significance of the differences between the groups was assessed using Student's $t$-test. Values of $p<0.05$ were considered statistically significant. The data were processed statistically using GraphPad Prism.

\subsubsection{Bacterial Biofilm Formation Inhibitory Activity}

Bacterial strains and cultivation conditions. For the detection of biofilms, formation strains Vibrio aquamarinus DSM 26054 and Acinetobacter calcoaceticus VKPM B-10353 were used. These strains form biofilms, making them useful for studying biofilms.

E. coli MG1655 (pRecA-lux) was used for the evaluation of the genotoxicity of the synthesized compounds. The biosensor with the PrecA promotor fixes the presence of the factors causing damage of DNA in a cell [74]. The biosensor E. coli MG1655 (pSoxS-lux) was used for the evaluation of prooxidant activity. The biosensor with the PsoxS promoter fixes the production of superoxide anion and NO [75]. Bioluminescent strains were obtained by the transformation of E. coli MG1655 by hybrid plasmids pRecA-lux, pSoxS-lux. The gene cassette luxCDABE Photorhabdus luminescens under the control PrecA promoters was used in this biosensor. This plasmid was created on the basis of pBR322 and contained a selective marker of ampicillin resistance (Amp gene). The strains were kindly furnished by Manukhov I.V., Federal State Unitary Enterprise "GosNIIGenetika").

The bacterial strains Acinetobacter calcoaceticus VKPM B-10353, E. coli MG1655 (pRecA-lux), and E. coli MG1655 (pSoxS-lux) were cultivated in Luria-Bertani (LB) medium [76] under constant shaking to early exponential phase at $37^{\circ} \mathrm{C}$. Cells were used immediately for stress induction tests. One hundred 
micrograms of ampicillin per milliliter were added into LB medium at cultivation of E. coli MG1655 (pRecA-lux) and E. coli MG1655 (pSoxS-lux). Strain V. aquamarinus DSM 26054 was grown in LB medium supplemented with $3 \% \mathrm{NaCl}$.

Chemicals. All of the chemicals used were of analytical grade. Crystal violet and $N$-methyl-N-nitro- $N$-nitrosoguanidine were obtained from Sigma-Aldrich (USA). Ampicillin was obtained from Sintez (Russia). Azithromycin was obtained from Farmstandart (Russia). Test solutions were prepared in deionized water immediately before the tests. Rat liver microsomal enzymes (S9 fraction) were from Moltox (USA).

The test compounds were dissolved in DMSO to the concentration of $1 \times 10^{-2} \mathrm{M}$. Then, they were diluted with ethanol. The control solutions were analogous dilutions of DMSO in ethanol. The tested compounds were also compared with the standard antibiotic azithromycin. Azithromycin was dissolved in DMSO to the concentration of $5 \times 10^{-3} \mathrm{M}$ and then diluted with deionized water.

Biosensors assay procedure. The detailed protocol of toxicity testing by means of a bacterial lux-biosensors is described in the article [77].

Calculation. The criterion of toxic influence was bioluminescence intensity change of the test object in the researched sample in comparison with the control sample.

The induction factor (I) was defined as the relation of luminescence intensity of a lux-biosensor suspension containing tested sample $\left(\mathrm{L}_{\mathrm{c}}\right)$ to the luminescence intensity of a lux-biosensor control suspension $\left(\mathrm{L}_{\mathrm{k}}\right)$ : $\mathrm{I}=\mathrm{L}_{\mathrm{c}} / \mathrm{L}_{\mathrm{k}}$.

If at significant differences from control induction factor values were $\leq 2$, the detected genotoxic effect was evaluated as "weak", if they were in the range from 2 to 10 as "medium", and above 10 as "strong". All the experiments were carried out three times independently.

Difference reliability of bioluminescence in experiment from control value was estimated by $t$-criterion with the help of Excel software. The conclusions about sample toxicity were made at $p<$ 0.05 .

Test system for evaluation of biofilms production. To quantify the formation of biofilms, the crystal violet assay was used, with some modifications [78]. The necessary concentrations of the test compounds were prepared as described above.

V. aquamarinus DSM 26054 was cultivated for $24 \mathrm{~h}$ in LB medium supplemented with $3 \% \mathrm{NaCl}$ in the Innova 40R shaker incubator (New Brunswick Scientific, USA) at $25^{\circ} \mathrm{C}$ and $200 \mathrm{rpm}$. A. calcoaceticus VKPM B-10353 was cultivated for $24 \mathrm{~h}$ in LB medium in the Innova 40R shaker incubator (New Brunswick Scientific, Enfield, CT, USA) at $30{ }^{\circ} \mathrm{C}$ and $200 \mathrm{rpm}$. Then, the suspensions of the daily culture of $V$. aquamarinus DSM 26054 and A. calcoaceticus VKPM B-10353 were diluted with LB medium supplemented with $3 \% \mathrm{NaCl}$ to the density of $1 \times 10^{8}$ cells $/ \mathrm{mL}$.

The resulting suspension $(180 \mu \mathrm{L})$ was added to the wells of a polystyrene microplate (Nuova Aptaca, Canelli, Italy). To some of the wells, $20 \mu \mathrm{L}$ of the test substances at various concentrations were added. Since solvents used could also influence the biofilm formation, $20 \mu \mathrm{L}$ of the appropriate solvent was added to the other part of the wells at same dilutions (control). Six replicates were done for each treatment and control. The microplate was covered with a lid and wrapped with Parafilm (Bemis Company, Inc., Oshkosh, WI, USA).

After incubation at $25^{\circ} \mathrm{C}$ for $72 \mathrm{~h}$, biofilms were stained. The contents in the wells were removed by means of a dispenser. The wells were then carefully washed three times with $250 \mu \mathrm{L}$ of sterile saline. The microplates were shaken to remove all non-adherent bacteria. Biofilms were fixed with $200 \mu \mathrm{L}$ of $96 \%$ ethanol for $15 \mathrm{~min}$. After the microplates had dried in air, $200 \mu \mathrm{L}$ of $0.5 \%$ crystal violet was introduced into the wells. After $10 \mathrm{~min}$, the dye was removed. The excess dye was removed by washing with water three times. After the microplates were air-dried, the dye in the wells bound to biofilms was dissolved with $200 \mu \mathrm{L}$ of $96 \%$ ethanol. The extraction level (absorption) of crystal violet by ethanol was measured after 60 minutes at a wavelength of $570 \mathrm{~nm}$ using a FLUOstar Omega microplate reader (BMG Labtech, Offenburg, Germany) in optical density units (OD570). The intensity of biofilm formation directly corresponds to the intensity of staining of the contents of the wells with 
the dye. Biofilm formation was determined by the difference between the mean OD readings obtained in the presence of compounds and the control.

Each experiment was performed in triplicate. The values were expressed as mean + SD. Student's $t$-test was used to compare these values. Differences were considered statistically significant at $p<0.05$.

\section{Conclusions}

In conclusion, a series of novel 2-(het)arylpyrrolidine-1-carboxamides were obtained via a modular approach based on intramolecular cyclization/Mannich-type reaction of $N$-(4,4-diethoxybutyl)ureas. Their anti-cancer activities were tested both in vitro and in vivo. A pyrrolidine derivative possessing a cyclo-hexyl substituent in the carboxamide moiety and a benzofuroxan fragment in the pyrrolidine ring was determined as the most active in the in vitro assay. Notably, its activity towards M-Hela tumor cell lines was found to be twice that of reference drug tamoxifen. At the same time, its cytotoxicity towards normal Chang liver cells did not exceed tamoxifen's toxicity. The obtained results indicate that the death of M-Hela cells presumably occurs via an apoptotic pathway due to activation of the surface cell receptors and not due to mitochondrial dysfunction. In the in vivo studies, water-soluble compounds possessing $\mathrm{N}$-(2-(dimethylamino)ethyl)pyrrolidine-1-carboxamide scaffold and either a heterocyclic (hydroxycoumarine) or aromatic (sesamol) substituent in the pyrrolidine core were proven to be the most effective. The number of surviving animals on day 60 of observation ranged from $17 \%$ to $83 \%$ and increased life span (ILS) ranged from $80 \%$ to $447 \%$. Additionally, compounds possessing a benzofuroxan moiety were found to effectively suppress bacterial biofilm growth, and thus are promising candidates for further development as anti-bacterial agents.

Supplementary Materials: The following are available online at http://www.mdpi.com/1420-3049/24/17/3086/s1, Figures S1-S14 (Anti-biofilm activity data), Figure S15, Tables S1-S4 (X-ray data); Figure S16 (In vivo anti-cancer activity data), copies of NMR spectra of all synthesized compounds.

Author Contributions: A.S.-investigation (chemistry), N.A.-investigation (chemistry), A.G.-supervision (chemistry), conceptualization, writing —original draft, E.C.—writing-review \& editing, funding acquisition, A.B.-project administration, I.S., A.G., S.K., M.Z.—investigation (anti-biofilm activity), M.S.- supervision (anti-biofilm activity), J.V.-investigation (X-ray study), A.V.- supervision (in vitro anti-cancer studies), A.S.-investigation (in vitro anti-cancer studies), E.K., T.S., U.A., A.B.-investigation (in vivo anti-cancer studies), D.M.-supervision (in vivo anti-cancer studies).

Funding: The reported study was funded by Russian Foundation for Basic Research (RFBR) according to the research project № 18-33-20023 and as part of the government assignment for the FRC Kazan Scientific Center of RAS. The study of anti-biofilm activity was funded by the Ministry of Education and Science of the Russian Federation (grant N 6.2379.2017/PCh), President of the Russian Federation (grant N NSh-3464.2018.11). The study of in vivo anti-cancer activity was funded by the Ministry of Education and Science of the Russian Federation (N 0089-2019-0014, 0089-2019-0016).

Acknowledgments: The authors are grateful to the Assigned Spectral-Analytical Center of FRC Kazan Scientific Center of RAS for technical assistance in research. The X-ray measurements were performed using shared experimental facilities supported by IGIC RAS state assignment.

Conflicts of Interest: The authors declare no conflicts of interest.

\section{References}

1. Talapatra, S.K.; Talapatra, B. Hygrine, hygroline, and cuscohygrine (ornithine-derived alkaloids) BT-Chemistry of plant natural products: Stereochemistry, conformation, synthesis, biology, and medicine. In Chemistry of Plant Natural Products; Talapatra, S.K., Talapatra, B., Eds.; Springer: Berlin/Heidelberg, Germany, 2015; pp. 725-732. ISBN 978-3-642-45410-3.

2. Carroll, F.I. Epibatidine analogs synthesized for characterization of nicotinic pharmacophores-A Review. Heterocycles 2009, 79, 99-120. [CrossRef]

3. Robertson, J.; Stevens, K. Pyrrolizidine alkaloids. Nat. Prod. Rep. 2014, 31, 1721-1788. [CrossRef] [PubMed]

4. Michael, J.P. Simple indolizidine and quinolizidine alkaloids. In Alkaloids: Chemistry and Biology; Academic Press: Cambridge, MA, USA, 2016; Volume 75, pp. 1-498. ISBN 9780128034347. 
5. Taylor, R.D.; MacCoss, M.; Lawson, A.D.G. Rings in drugs. J. Med. Chem. 2014, 57, 5845-5859. [CrossRef] [PubMed]

6. Hollstein, U. Actinomycin. Chemistry and mechanism of action. Chem. Rev. 1974, 74, 625-652. [CrossRef]

7. Byrd, J.C.; Harrington, B.; O’Brien, S.; Jones, J.A.; Schuh, A.; Devereux, S.; Chaves, J.; Wierda, W.G.; Awan, F.T.; Brown, J.R.; et al. Acalabrutinib (ACP-196) in relapsed chronic lymphocytic leukemia. N. Engl. J. Med. 2016, 374, 323-332. [CrossRef]

8. Wu, J.; Zhang, M.; Liu, D. Acalabrutinib (ACP-196): A selective second-generation BTK inhibitor. J. Hematol. Oncol. 2016, 9, 21. [CrossRef]

9. DuBois, S.G.; Laetsch, T.W.; Federman, N.; Turpin, B.K.; Albert, C.M.; Nagasubramanian, R.; Anderson, M.E.; Davis, J.L.; Qamoos, H.E.; Reynolds, M.E.; et al. The use of neoadjuvant larotrectinib in the management of children with locally advanced TRK fusion sarcomas. Cancer 2018, 124, 4241-4247. [CrossRef]

10. Ziegler, D.S.; Wong, M.; Mayoh, C.; Kumar, A.; Tsoli, M.; Mould, E.; Tyrrell, V.; Khuong-Quang, D.-A.; Pinese, M.; Gayevskiy, V.; et al. Brief Report: Potent clinical and radiological response to larotrectinib in TRK fusion-driven high-grade glioma. Br. J. Cancer 2018, 119, 693-696. [CrossRef]

11. Jemal, A.; Bray, F.; Center, M.M.; Ferlay, J.; Ward, E.; Forman, D. Global cancer statistics. CA Cancer J. Clin. 2011, 61, 69-90. [CrossRef]

12. Le, C.; Liang, Y.; Evans, R.W.; Li, X.; MacMillan, D.W.C. Selective sp3 C-H alkylation via polarity-match-based cross-coupling. Nature 2017, 547, 79-83. [CrossRef]

13. Deng, X.; Lei, X.; Nie, G.; Jia, L.; Li, Y.; Chen, Y. Copper-catalyzed cross-dehydrogenative N 2 -coupling of $\mathrm{NH}-1,2,3$-Triazoles with N, N-Dialkylamides: N-Amidoalkylation of NH-1,2,3-Triazoles. J. Org. Chem. 2017, 82, 6163-6171. [CrossRef] [PubMed]

14. Angibaud, P.R.; Querolle, O.A.G.; Berthelot, D.J.-C.; Meyer, C.; Willot, M.P.V.; Meerpoel, L. Preparation of quinoxaline and pyridopyrazine derivatives as pI3K $\beta$ inhibitors. Intnl. Patent Appl. WO 2017060406, 13 April 2017.

15. Barlaam, B.; Cosulich, S.; Degorce, S.; Ellston, R.; Fitzek, M.; Green, S.; Hancox, U.; Lambert-van der Brempt, C.; Lohmann, J.-J.; Maudet, M.; et al. Discovery of a series of 8-(1-phenylpyrrolidin-2-yl)-6-carboxamide-2-morpholino-4H-chromen-4-one as PI3K $\beta / \delta$ inhibitors for the treatment of PTEN-deficient tumours. Bioorg. Med. Chem. Lett. 2017, 27, 1949-1954. [CrossRef] [PubMed]

16. Mu, X.; Shibata, Y.; Makida, Y.; Fu, G.C. Control of vicinal stereocenters through nickel-catalyzed alkyl-alkyl cross-coupling. Angew. Chemie Int. Ed. 2017, 56, 5821-5824. [CrossRef] [PubMed]

17. Park, Y.; Schindler, C.S.; Jacobsen, E.N. Enantioselective Aza-Sakurai Cyclizations: Dual role of thiourea as H-bond donor and Lewis base. J. Am. Chem. Soc. 2016, 138, 14848-14851. [CrossRef] [PubMed]

18. He, J.; Dhakshinamoorthy, A.; Primo, A.; Garcia, H. Iron nanoparticles embedded in graphitic carbon matrix as heterogeneous catalysts for the oxidative $\mathrm{C}-\mathrm{N}$ coupling of aromatic $\mathrm{N}-\mathrm{H}$ compounds and amides. ChemCatChem 2017, 9, 3003-3012. [CrossRef]

19. Dian, L.; Zhang-Negrerie, D.; Du, Y. Transition metal-free oxidative cross-coupling C(sp 2)-C(sp 3) bond formation: Regioselective C-3 alkylation of coumarins with tertiary amines. Adv. Synth. Catal. 2017, 359, 3090-3094. [CrossRef]

20. Panda, S.; Coffin, A.; Nguyen, Q.N.; Tantillo, D.J.; Ready, J.M. Synthesis and Utility of Dihydropyridine Boronic Esters. Angew. Chemie Int. Ed. 2016, 55, 2205-2209. [CrossRef]

21. Xie, J.; Rudolph, M.; Rominger, F.; Hashmi, A.S.K. Photoredox-controlled mono- and Di-multifluoroarylation of C(sp 3 )-H bonds with aryl fluorides. Angew. Chemie Int. Ed. 2017, 56, 7266-7270. [CrossRef]

22. Kamijo, S.; Kamijo, K.; Murafuji, T. Synthesis of alkylated pyrimidines via photoinduced coupling using benzophenone as a mediator. J. Org. Chem. 2017, 82, 2664-2671. [CrossRef]

23. Vega, J.A.; Alonso, J.M.; Méndez, G.; Ciordia, M.; Delgado, F.; Trabanco, A.A. Continuous flow $\alpha$-Arylation of N, N-Dialkylhydrazones under visible-light photoredox catalysis. Org. Lett. 2017, 19, 938-941. [CrossRef]

24. Ahneman, D.T.; Doyle, A.G. C-H functionalization of amines with aryl halides by nickel-photoredox catalysis. Chem. Sci. 2016, 7, 7002-7006. [CrossRef] [PubMed]

25. Shaw, M.H.; Shurtleff, V.W.; Terrett, J.A.; Cuthbertson, J.D.; MacMillan, D.W.C. Native functionality in triple catalytic cross-coupling: Sp3 C-H bonds as latent nucleophiles. Science 2016, 352, 1304-1308. [CrossRef] [PubMed]

26. Shih, Y.-C.; Wang, J.-S.; Hsu, C.-C.; Tsai, P.-H.; Chien, T.-C. Identification of reactive intermediates for the decarbonylative reaction of 1-alkylprolines. Synlett 2016, 27, 2841-2845. 
27. Cheng, W.-M.; Shang, R.; Fu, Y. Photoredox/Brønsted acid co-catalysis enabling decarboxylative coupling of amino acid and peptide redox-active esters with N-heteroarenes. ACS Catal. 2017, 7, 907-911. [CrossRef]

28. Lipp, B.; Nauth, A.M.; Opatz, T. Transition-metal-free decarboxylative photoredox coupling of carboxylic acids and alcohols with aromatic nitriles. J. Org. Chem. 2016, 81, 6875-6882. [CrossRef] [PubMed]

29. Luo, J.; Zhang, J. Donor-Acceptor fluorophores for visible-light-promoted organic synthesis: Photoredox/Ni dual catalytic C (sp 3)-C (sp 2) cross-coupling. ACS Catal. 2016, 6, 873-877. [CrossRef]

30. Lovett, G.H.; Sparling, B.A. Decarboxylative anti-michael addition to olefins mediated by photoredox catalysis. Org. Lett. 2016, 18, 3494-3497. [CrossRef]

31. Baudelet, D.; Daïch, A.; Rigo, B.; Lipka, E.; Gautret, P.; Homerin, G.; Claverie, C.; Rousseau, J.; Abuhaie, C.-M.; Ghinet, A. Impact of functional groups on the copper-initiated N-arylation of 5-functionalized pyrrolidin-2-ones and their vinylogues. Synthesis (Stuttg) 2016, 48, 2226-2244. [CrossRef]

32. Yamashita, Y.; Nam, L.C.; Dutton, M.J.; Yoshimoto, S.; Kobayashi, S. Catalytic asymmetric endo-selective [3+ 2] cycloaddition reactions of Schiff bases of $\alpha$-aminophosphonates with olefins using chiral metal amides. Chem. Commun. (Cambridge, United Kingdom) 2015, 51, 17064-17067. [CrossRef]

33. Yamashita, Y.; Imaizumi, T.; Guo, X.-X.; Kobayashi, S. Chiral Silver Amides as Effective Catalysts for Enantioselective [3 + 2] Cycloaddition Reactions. Chem. Asian J. 2011, 6, 2550-2559. [CrossRef]

34. Yamashita, Y.; Guo, X.-X.; Takashita, R.; Kobayashi, S. Chiral silver amide-catalyzed enantioselective [3 + 2] cycloaddition of $\alpha$-aminophosphonates with olefins. J. Am. Chem. Soc. 2010, 132, 3262-3263. [CrossRef] [PubMed]

35. Dondas, H.A.; Durust, Y.; Grigg, R.; Slater, M.J.; Sarker, M.A.B. XYZH systems as potential 1,3-dipoles. Part 62: 1,3-dipolar cycloaddition reactions of metallo-azomethine ylides derived from $\alpha$-iminophosphonates. Tetrahedron 2005, 61, 10667-10682. [CrossRef]

36. Feng, B.; Chen, J.-R.; Yang, Y.-F.; Lu, B.; Xiao, W.-J. A highly enantioselective copper/phosphoramidite-thioether-catalyzed diastereodivergent 1,3-dipolar cycloaddition of azomethine ylides and nitroalkenes. Chem. A Eur. J. 2018, 24, 1714-1719. [CrossRef] [PubMed]

37. Ponce, A.; Alonso, I.; Adrio, J.; Carretero, J.C. Stereoselective Ag-catalyzed 1,3-dipolar cycloaddition of activated trifluoromethyl-substituted azomethine ylides. Chem. A Eur. J. 2016, 22, 4952-4959. [CrossRef] [PubMed]

38. Gazizov, A.S.; Smolobochkin, A.V.; Burilov, A.R.; Pudovik, M.A. Interaction of 2-naphthol with $\gamma$-ureidoacetals. A new method for the synthesis of 2-arylpyrrolidines. Chem. Heterocycl. Compd. 2014, 50, 707-714. [CrossRef]

39. Gazizov, A.S.; Smolobochkin, A.V.; Voronina, J.K.; Burilov, A.R.; Pudovik, M.A. Acid-catalyzed ring opening in 2-(2-hydroxynaphthalene-1-yl)-pyrrolidine-1-carboxamides: Formation of dibenzoxanthenes, diarylmethanes, and calixarenes. Tetrahedron 2015, 71, 445-450. [CrossRef]

40. Gazizov, A.S.; Kharitonova, N.I.; Smolobochkin, A.V.; Syakaev, V.V.; Burilov, A.R.; Pudovik, M.A. Facile synthesis of 2-(2-arylpyrrolidin-1-yl)pyrimidines via acid-catalyzed reaction of N-(4,4-diethoxybutyl)pyrimidin-2-amine with phenols. Monat. Chem. 2015, 146, 1845-1849. [CrossRef]

41. Gazizov, A.S.; Burilov, A.R.; Pudovik, M.A. Reactions of polyhydric phenols with nitrogen-containing acetals in the synthesis of polyphenols and heterocyclic compounds. Russ. Chem. Bull. 2016, 65, 2143-2150. [CrossRef]

42. Smolobochkin, A.V.; Gazizov, A.S.; Syakaev, V.V.; Anikina, E.A.; Burilov, A.R.; Pudovik, M.A. Synthesis of 2-arylpyrrolidine-1-carboxamides via acid-catalyzed reaction of (4,4-diethoxybutyl)ureas with 3-aminophenol. Monat. Chem. 2017, 148, 1433-1438. [CrossRef]

43. Smolobochkin, A.V.; Gazizov, A.S.; Anikina, E.A.; Burilov, A.R.; Pudovik, M.A. Acid-catalyzed reaction of phenols with N-(4,4-diethoxybutyl) sulfonamides-A new method for the synthesis of 2-aryl-1-sulfonylpyrrolidines. Chem. Heterocycl. Compd. 2017, 53, 161-166. [CrossRef]

44. Gazizov, A.S.; Smolobochkin, A.V.; Anikina, E.A.; Voronina, J.K.; Burilov, A.R.; Pudovik, M.A. Acid-catalyzed intramolecular cyclization of N-(4,4-diethoxybutyl) sulfonamides as a novel approach to the 1-sulfonyl-2-arylpyrrolidines. Synth. Commun. 2017, 47, 44-52. [CrossRef]

45. Smolobochkin, A.V.; Gazizov, A.S.; Burilov, A.R.; Pudovik, M.A. Synthesis of functionalized diarylbutane derivatives by the reaction of 2-methylresorcinol with $\gamma$-ureidoacetals. Russ. J. Gen. Chem. 2015, 85, 1779-1782. [CrossRef] 
46. Gazizov, A.S.; Smolobochkin, A.V.; Voronina, Y.K.; Burilov, A.R.; Pudovik, M.A. New method of synthesis of 2-arylpyrrolidines: Reaction of resorcinol and its derivatives with $\gamma$-ureidoacetals. Arkivoc 2014, 2014, 319-327.

47. Huang, L. Impact of solid state properties on developability assessment of drug candidates. Adv. Drug Deliv. Rev. 2004, 56, 321-334. [CrossRef] [PubMed]

48. Corrigan, O.I. Salt forms: Pharmaceutical aspects. In Encyclopedia of Pharmaceutical Technology; Swarbrick, J., Ed.; Informa Healthcare: New York, NY, USA, 2006; pp. 3177-3187.

49. Kim, J.Y.; Choi, D.S.; Jung, M.Y. Antiphoto-oxidative activity of sesamol in methylene blue- and chlorophyll-sensitized photo-oxidation of oil. J. Agric. Food Chem. 2003, 51, 3460-3465. [CrossRef]

50. Fukuda, Y.; Nagata, M.; Osawa, T.; Namiki, M. Contribution of lignan analogues to antioxidative activity of refined unroasted sesame seed oil. J. Am. Oil Chem. Soc. 1986, 63, 1027-1031. [CrossRef]

51. Zhang, M.-Z.; Zhang, R.-R.; Wang, J.-Q.; Yu, X.; Zhang, Y.-L.; Wang, Q.-Q.; Zhang, W.-H. Microwave-assisted synthesis and antifungal activity of novel fused osthole derivatives. Eur. J. Med. Chem. 2016, 124, 10-16. [CrossRef]

52. Molodtsov, V.; Fleming, P.R.; Eyermann, C.J.; Ferguson, A.D.; Foulk, M.A.; McKinney, D.C.; Masse, C.E.; Buurman, E.T.; Murakami, K.S. X-ray crystal structures of Escherichia coli RNA polymerase with switch region binding inhibitors enable rational design of squaramides with an improved fraction unbound to human plasma protein. J. Med. Chem. 2015, 58, 3156-3171. [CrossRef]

53. Fang, Z.; Liao, P.-C.; Yang, Y.-L.; Yang, F.-L.; Chen, Y.-L.; Lam, Y.; Hua, K.-F.; Wu, S.-H. Synthesis and biological evaluation of polyenylpyrrole derivatives as anticancer agents acting through caspases-dependent apoptosis. J. Med. Chem. 2010, 53, 7967-7978. [CrossRef]

54. Chugunova, E.; Akylbekov, N.; Shakirova, L.; Dobrynin, A.; Syakaev, V.; Latypov, S.; Bukharov, S.; Burilov, A. Synthesis of hybrids of benzofuroxan and N-, S-containing sterically hindered phenols derivatives. Tautomerism. Tetrahedron 2016, 72, 6415-6420. [CrossRef]

55. Chugunova, E.A.; Voloshina, A.D.; Mukhamatdinova, R.E.; Serkov, I.V.; Proshin, A.N.; Gibadullina, E.M.; Burilov, A.R.; Kulik, N.V.; Zobov, V.V.; Krivolapov, D.B.; et al. The study of the biological activity of amino-substituted benzofuroxans. Lett. Drug Des. Discov. 2014, 11, 502-512. [CrossRef]

56. Chugunova, E.A.; Sazykina, M.A.; Gibadullina, E.M.; Burilov, A.R.; Sazykin, I.S.; Chistyakov, V.A.; Timasheva, R.E.; Krivolapov, D.B.; Goumont, R. Synthesis, genotoxicity and uv-protective activity of new benzofuroxans substituted by aromatic amines. Lett. Drug Des. Discov. 2013, 10, 145-154. [CrossRef]

57. Schiefer, I.T.; VandeVrede, L.; Fa', M.; Arancio, O.; Thatcher, G.R.J. Furoxans (1,2,5-oxadiazole- N -oxides) as novel NO mimetic neuroprotective and procognitive agents. J. Med. Chem. 2012, 55, 3076-3087. [CrossRef] [PubMed]

58. Gasco, A.; Fruttero, R.; Sorba, G.; Di Stilo, A.; Calvino, R. NO donors: Focus on furoxan derivatives. Pure Appl. Chem. 2004, 76, 973-981. [CrossRef]

59. Teicher, B.A. (Ed.) Tumor Models in Cancer Research; Humana Press: Totowa, NJ, USA, 2011; ISBN 978-1-60761-967-3.

60. Mironov, A.N.; Bunyatyan, N.D. (Eds.) Handbook for Preclinical Drug Trials; Ministry of Public Health and Social Development of the RF, FGBU Scientific Centre for the Expert Evaluation of Medicinal Products: Moscow, Russia, 2012.

61. Rabin, N.; Zheng, Y.; Opoku-Temeng, C.; Du, Y.; Bonsu, E.; Sintim, H.O. Biofilm formation mechanisms and targets for developing antibiofilm agents. Future Med. Chem. 2015, 7, 493-512. [CrossRef] [PubMed]

62. Rittmann, B.E. Biofilms, active substrata, and me. Water Res. 2018, 132, 135-145. [CrossRef] [PubMed]

63. Hobley, L.; Harkins, C.; MacPhee, C.E.; Stanley-Wall, N.R. Giving structure to the biofilm matrix: An overview of individual strategies and emerging common themes. FEMS Microbiol. Rev. 2015, 39, 649-669. [CrossRef] [PubMed]

64. Hall, M.R.; McGillicuddy, E.; Kaplan, L.J. Biofilm: Basic principles, pathophysiology, and implications for clinicians. Surg. Infect. (Larchmt). 2014, 15, 1-7. [CrossRef]

65. Jamal, M.; Ahmad, W.; Andleeb, S.; Jalil, F.; Imran, M.; Nawaz, M.A.; Hussain, T.; Ali, M.; Rafiq, M.; Kamil, M.A. Bacterial biofilm and associated infections. J. Chin. Med. Assoc. 2018, 81, 7-11. [CrossRef]

66. Peng, J.-S.; Tsai, W.-C.; Chou, C.-C. Inactivation and removal of Bacillus cereus by sanitizer and detergent. Int. J. Food Microbiol. 2002, 77, 11-18. [CrossRef]

67. Goldberg, J. Biofilms and antibiotic resistance: A genetic linkage. Trends Microbiol. 2002, 10, 264. [CrossRef] 
68. Elinav, E.; Nowarski, R.; Thaiss, C.A.; Hu, B.; Jin, C.; Flavell, R.A. Inflammation-induced cancer: Crosstalk between tumours, immune cells and microorganisms. Nat. Rev. Cancer 2013, 13, 759-771. [CrossRef] [PubMed]

69. Rao, C.; Verma, A.; Gupta, P.; Vijayakumar, M. Anti-inflammatory and anti-nociceptive activities of Fumaria indica whole plant extract in experimental animals. Acta Pharm. 2007, 57, 491-498. [CrossRef] [PubMed]

70. Samanta, A.; Podder, S.; Kumarasamy, M.; Ghosh, C.K.; Lahiri, D.; Roy, P.; Bhattacharjee, S.; Ghosh, J.; Mukhopadhyay, A.K. Au nanoparticle-decorated aragonite microdumbbells for enhanced antibacterial and anticancer activities. Mater. Sci. Eng. C 2019, 103, 109734. [CrossRef] [PubMed]

71. Sheldrick, G.M. SHELXTL v.6.12, Structure Determination Software Suite; Bruker AXS: Madison, WI, USA, 2000.

72. Dolomanov, O.V.; Bourhis, L.J.; Gildea, R.J.; Howard, J.A.K.; Puschmann, H. OLEX2: A complete structure solution, refinement and analysis program. J. Appl. Crystallogr. 2009, 42, 339-341. [CrossRef]

73. Macrae, C.F.; Bruno, I.J.; Chisholm, J.A.; Edgington, P.R.; McCabe, P.; Pidcock, E.; Rodriguez-Monge, L.; Taylor, R.; van de Streek, J.; Wood, P.A. Mercury CSD 2.0-New features for the visualization and investigation of crystal structures. J. Appl. Crystallogr. 2008, 41, 466-470. [CrossRef]

74. Vollmer, A.C.; Belkin, S.; Smulski, D.R.; Van Dyk, T.K.; LaRossa, R.A. Detection of DNA damage by use of Escherichia coli carrying recA'::lux, uvrA'::lux, or alkA'::lux reporter plasmids. Appl. Environ. Microbiol. 1997, 63, 2566-2571.

75. Lushchak, V.I. Adaptive response to oxidative stress: Bacteria, fungi, plants and animals. Comp. Biochem. Physiol. Part C Toxicol. Pharmacol. 2011, 153, 175-190. [CrossRef]

76. Maniatis, T.; Fritsch, E.F.F.; Sambrook, J.; Fritsch, E.F.F.; Maniatis, T. Molecular Cloning: A Laboratory Manual; Cold Spring Harbor Laboratory Press: New York, NY, USA, 1982; ISBN 0-87969-136-0.

77. Chugunova, E.; Boga, C.; Sazykin, I.; Cino, S.; Micheletti, G.; Mazzanti, A.; Sazykina, M.; Burilov, A.; Khmelevtsova, L.; Kostina, N. Synthesis and antimicrobial activity of novel structural hybrids of benzofuroxan and benzothiazole derivatives. Eur. J. Med. Chem. 2015, 93, 349-359. [CrossRef]

78. Stepanović, S.; Vuković, D.; Dakić, I.; Savić, B.; Švabić-Vlahović, M. A modified microtiter-plate test for quantification of staphylococcal biofilm formation. J. Microbiol. Methods 2000, 40, 175-179. [CrossRef]

Sample Availability: Samples of the compounds 4, 5-8 are available from the authors.

(C) 2019 by the authors. Licensee MDPI, Basel, Switzerland. This article is an open access article distributed under the terms and conditions of the Creative Commons Attribution (CC BY) license (http://creativecommons.org/licenses/by/4.0/). 